\title{
Comparison principle for a Generalized Fast Marching Method
}

\author{
Nicolas Forcadel ${ }^{1,2}$
}

March 20, 2008

\begin{abstract}
In [4], the authors have proposed a generalization of the classical Fast Marching Method of Sethian for the eikonal equation in the case where the normal velocity depends on space and time and can change sign. The goal of this paper is to propose a modified version of the Generalized Fast Marching Method proposed in [4] for which we state a general comparison principle. We also prove the convergence of the new algorithm.
\end{abstract}

AMS Classification: 65M06, 65M12, 49L25 .

Keywords: Eikonal equation, fast marching scheme, monotone scheme, convergence.

\section{Introduction}

In this paper, we are interested in the Fast Marching Method (FMM). This method has been introduced by Sethian [8, 9] (see also Tsitsiklis [10] for a previous single-pass method) and adapted for general given normal velocity $c(x, t)$ in [4] (we refer to [4] for a detailed discussion and for more references on Fast Marching Method). More precisely, in [4] the authors have proposed a generalization of the classical FMM for the following equation

$$
\left\{\begin{array}{lll}
\theta_{t}=c(x, t)|D \theta| & \text { in } & \mathbb{R}^{N} \times(0,+\infty), \\
\theta(\cdot, 0)=1_{\Omega_{0}}-1_{\Omega_{0}^{c}} & \text { on } & \mathbb{R}^{N}
\end{array}\right.
$$

and we have proved a convergence result in the class of discontinuous viscosity solutions for our Generalized Fast Marching Method (GFMM). A major improvement of our GFMM is that it can deal with a velocity changing sign. A drawback of this algorithm is that it was not completely monotone (see Subsection 5.2). In (1.1) and throughout the paper, $\theta_{t}$ denotes the time derivative of $\theta$ and $D \theta$ its gradient in space.

The goal of this paper is to propose a GFMM slightly different from the one developed in [4], which is now truly monotone (see the comparison principle Theorem 3.4). The monotony of a scheme is a very important property. Indeed it is a key ingredient to prove the convergence of the scheme (see the general proof of convergence of Barles and Souganidis [3]), but it can also be used to prove some error estimate (we refer to Crandall, Lions [6]). In this direction, we give some heuristic error estimate in Theorem 8.1. We will explore more carefully the rate of convergence of our GFMM in a future work.

The comparison principle can also be useful to prove convergence result for non-local dynamics. We will use, in particular, this algorithm in a future work [5] to study numerically the dynamics of one dislocation line (see Alvarez, Hoch, Le Bouar, Monneau [2] for a description of the mathematical model).

\footnotetext{
${ }^{1}$ Projet Commands, CMAP-INRIA Futurs, Ecole Polytechnique, 91128 Palaiseau and ENSTA, UMA, 32 Bd Victor, 75739 Paris Cedex 15, France.

${ }^{2}$ CERMICS, Paris Est-ENPC, 6 \& 8 avenue Blaise Pascal, Cité Descartes, Champs sur Marne, 77455 Marne la Vallée Cedex 2, France.
} 


\section{Organization of the paper}

In Section 2, we give the modified version of the GFMM. The main results are presented in Section 3. In Section 4, we give some general properties of the algorithm. Sections 5 and 6 are devoted to the proof of the comparison principle Theorem 3.4. We also give a counter-example for the GFMM of [4]. In Section 7, we prove the convergence of the algorithm (see Theorem 3.6) and we provide an heuristic error estimate (Theorem 8.1).

\section{The monotone GFMM algorithm}

We begin this section by describing the new feature of our algorithm which makes it monotone.

Let us first recall the basic ideas of the classical FMM (as well as the GFMM of [4]). One feature of the classical FMM is to generate a sequence of times $t_{n}$. In the GFMM algorithm (and also in the FMM) we also need a phase parameter $\theta_{I}^{n}$ with values +1 or -1 (which is in fact represented by the frozen points and its complementary set in the classical FMM) defined at each step $n$ of the algorithm and for any $I \in \mathbb{Z}^{N}$. This $\theta_{I}^{n}$ should be thought as a discretisation of the solution $\theta$ of equation (1.1) at time $t_{n}$ and at point $x_{I}=I \Delta x(\Delta x$ being the space step).

To make evolve the front (i.e. the discontinuity of $\theta_{I}^{n}$ ), the FMM also needs to introduce a time $u_{I}^{n}$ defined for $I$ in the whole front at the $n$-th iteration of the algorithm. This time $u_{I}^{n}$ can be interpreted in the classical FMM as the time when the front reaches the point $I$ (this interpretation is essentially true for our GFMM algorithm except in the more delicate case where the velocity vanishes). The goal is then to find the next point reached by the front. To do this, the idea is to compute a tentative value $\tilde{u}_{I}^{n}$ (at the $n$-th iteration and at the point $x_{I}$ ) of the arrival time for all points which can be immediately reached by the front (which is called the Narrow Band). The computation of the tentative value $\tilde{u}_{I}^{n}$ uses the time $u_{J}^{n}$ for $J$ in the neighbourhood of $I$. The next points reached by the front is then the ones which have the minimum tentative value. We then denote by $t_{n+1}$ this minimum value and we accept (i.e. we change the $\theta$ ) at time $t_{n+1}$ the points that realize the minimum and we iterate.

In the case of positive velocity, it has been proved that the GFMM scheme of [4] is monotone (as well as the classical FMM). This is not the case when the velocity can change sign (see the counter example in Subsection 5.2). This essentially comes from the change of the sign in time of the velocity. Indeed, to compute the tentative value $\tilde{u}_{I}^{n}$, when the velocity changes sign, the good notion to use is the time when the front begins to go from a neighbour $J$ of $I$ to $I$, and not the time when the front reaches the point $J$. This two notions are the same when the velocity is of constant sign in time but they differ when the velocity changes sign. Moreover, for a point $J$, the time when the front begins to go from $J$ to each direction can be different. This is the reason why we have to introduce several times for each node, in fact a time for each direction (see the details of the algorithm in Section 2).

With this new notion, we recover a good interpretation of the computation (even when the velocity vanishes or changes sign) and it is in fact sufficient to recover the monotonicity of the scheme.

\subsection{Preliminaries}

We consider a modified version of the GFMM algorithm introduced in [4].

Let us consider a lattice $Q=\left\{x_{I}=\left(x_{i_{1}}, . ., x_{i_{N}}\right)=\left(i_{1} \Delta x, . ., i_{N} \Delta x\right), I=\left(i_{1}, . ., i_{N}\right) \in \mathbb{Z}^{N}\right\}$ with space step $\Delta x$. We will also use a time step $\Delta t>0$ (which does not satisfy any CFL condition).

The following definitions will be useful in the following.

Definition 2.1 The neighbourhood of the node $I \in \mathbb{Z}^{N}$ is the set

$$
V(I) \equiv\left\{J \in \mathbb{Z}^{N}:|J-I| \leq 1\right\} .
$$

Definition 2.2 Given the speed $c_{I}^{n} \equiv c\left(x_{I}, t_{n}\right)$ we define the function

$$
\widehat{c}_{I}^{n} \equiv \begin{cases}0 & \text { if there exists } J \in V(I) \text { such that }\left(c_{I}^{n} c_{J}^{n}<0 \text { and }\left|c_{I}^{n}\right| \leq\left|c_{J}^{n}\right|\right), \\ c_{I}^{n} & \text { otherwise. }\end{cases}
$$

Definition 2.3 The numerical boundary $\partial E$ of a set $E \subset \mathbb{Z}^{N}$ is

$$
\partial E \equiv V(E) \backslash E
$$


with

$$
V(E)=\left\{J \in \mathbb{Z}^{N}, \quad \exists I \in E, \quad J \in V(I)\right\}
$$

Definition 2.4 Given a field $\theta^{n}: \mathbb{Z}^{N} \rightarrow\{+1,-1\}$, we define the two phases

$$
\Theta_{ \pm}^{n} \equiv\left\{I: \theta_{I}^{n}= \pm 1\right\}
$$

and the fronts

$$
F_{ \pm}^{n} \equiv \partial \Theta_{\mp}^{n}, \quad F^{n} \equiv F_{+}^{n} \cup F_{-}^{n} .
$$

We now describe our modified GFMM algorithm for unsigned velocity. As in the classical FMM, we define the Narrow Band (NB) which consists on the points $I \in \mathbb{Z}^{N}$ that can be immediately reached by the front:

$$
N B^{n}=\left\{I \in \mathbb{Z}^{N}, \exists J \in V(I), \theta_{I}^{n}=-\theta_{J}^{n} \text { and } \theta_{I}^{n} \hat{c}_{I}^{n}<0\right\}, \quad N B_{ \pm}^{n}=N B^{n} \cap\left\{I, \theta_{I}^{n}= \pm 1\right\}
$$

We observe that the Narrow Bands $N B_{ \pm}^{n}$ are related with the previous definition of fronts set as following:

$$
N B_{+}^{n}=F_{+}^{n} \cap\left\{I, \hat{c}_{I}^{n}<0\right\}, \quad N B_{-}^{n}=F_{-}^{n} \cap\left\{I, \hat{c}_{I}^{n}>0\right\} .
$$

Fig.1 shows the Front and the Narrow Band, the white part of the picture represents the domain where the speed is negative and the grey part the domain with speed positive.

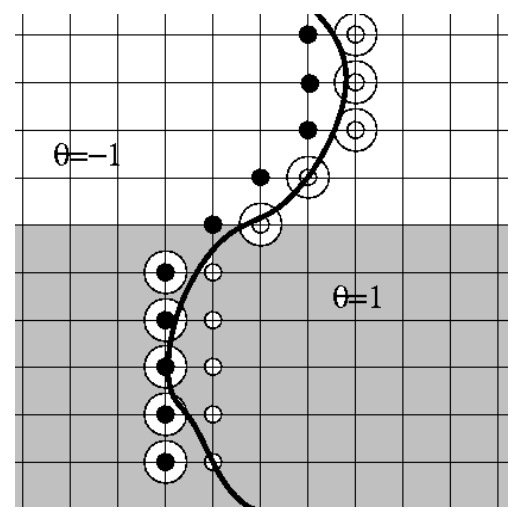

Figure 1: The Front $F^{+}$(white spot), the front $F^{-}$(black spot), the Narrow Band $N B^{+}$(circled white spot) and the Narrow Band $N B^{-}$(circled black spot). In the white domain the speed is negative, in the grey domain the speed is positive.

As in the FMM, for all $I \in N B^{n}$, we have to compute a tentative value (which we denote $\tilde{u}_{I}^{n}$ in the sequel) of the arrival time of the front at point $I$. To compute this tentative value, we define the points that are useful for $I$, i.e. that we will use in the computation of $\tilde{u}_{I}^{n}$ :

$$
\mathcal{U}^{n}(I)=\left\{J \in V(I), \theta_{I}^{n}=-\theta_{J}^{n}\right\}, \quad \mathcal{U}^{n}=\cup_{I \in N B^{n}} \mathcal{U}^{n}(I) .
$$

For all the points $J$ that are useful for a point $I \in N B^{n}$ (i.e. $J \in \mathcal{U}^{n}(I)$ ) we will introduce a time $u_{J \rightarrow I}^{n}$. This time $u_{J \rightarrow I}^{n}$ can be interpreted as the time when the front $F^{n}$ begin to go from point $J$ to point $I$ and will be used to compute the tentative value at point $I$.

The algorithm is now very similar to the classical Fast Marching Method. Once we have computed the tentative values for all points of the Narrow Band, we denote by $\tilde{t}_{n}$ the minimum of all this values. Unfortunately, this sequence of "candidate" time is not non-decreasing, so we have to truncate $\tilde{t}_{n}$ to define $t_{n}$ such that (see Step 6 of the algorithm):

$$
0 \leq t_{n}-t_{n-1} \leq \Delta t
$$

for a fixed $\Delta t$ (independent on $\Delta x$ ). We then accept (in most of the cases, see Step 7 of the algorithm) all the points that realize the minimum (i.e., we change the value of the $\theta$ ), we redefine the values $u_{J \rightarrow I}^{n}$ and we iterate. 


\subsection{The algorithm step-by-step}

We now give the details of the algorithm:

\section{Initialization}

1. Set $n=1$

2. Initialize the field $\theta^{0}$ as

$\theta_{I}^{0}= \begin{cases}1 & \text { for } x_{I} \in \Omega_{0} \\ -1 & \text { elsewhere }\end{cases}$

3. Initialize the time for points I

$$
u_{I \rightarrow K}^{0}= \begin{cases}t_{0} & \text { if } I \in \mathcal{U}^{0}(K) \text { and } K \in N B^{0} \\ +\infty & \text { otherwise }\end{cases}
$$

\section{Loop}

4. Compute $\tilde{u}^{n-1}$ on $N B^{n-1}$

Let $I \in N B^{n-1}$, then we compute $\tilde{u}_{I}^{n-1}$ as the solution of the following second order equation:

$$
\sum_{k=1}^{N}\left(\max _{ \pm}\left(0, \tilde{u}_{I}^{n-1}-u_{I^{k, \pm} \rightarrow I}^{n-1}\right)\right)^{2}=\frac{(\Delta x)^{2}}{\left|\widehat{c}_{I}^{n-1}\right|^{2}}
$$

where

$$
I^{k, \pm}=\left(i_{1}, . ., i_{k-1}, i_{k} \pm 1, i_{k+1}, . ., i_{N}\right) .
$$

5. $\tilde{t}_{n}=\min \left\{\tilde{u}_{I}^{n-1}, I \in N B^{n-1}\right\}$.

6. Truncate $\tilde{t}_{n}$ $t_{n}=\max \left(t_{n-1}, \min \left\{\tilde{t}_{n}, t_{n-1}+\Delta t\right\}\right)$

7. if $t_{n}=t_{n-1}+\Delta t$ and $t_{n}<\tilde{t}_{n}$ go to 4 with $n:=n+1, \theta^{n}=\theta^{n-1}$ and $u^{n}=u^{n-1}$.

8. Initialize the new accepted points $N A_{ \pm}^{n}=\left\{I \in N B_{ \pm}^{n-1}, \tilde{u}_{I}^{n-1}=\tilde{t}_{n}\right\}, N A^{n}=N A_{+}^{n} \cup N A_{-}^{n}$

9. Reinitialize $\theta^{n}$

$$
\theta_{I}^{n}= \begin{cases}-\theta_{I}^{n-1} & \text { for } I \in N A^{n} \\ \theta_{I}^{n-1} & \text { otherwise }\end{cases}
$$

10. Reinitialize $u_{I \rightarrow K}^{n}$

$$
u_{I \rightarrow K}^{n}= \begin{cases}\min \left(u_{I \rightarrow K}^{n-1}, t_{n}\right) & \text { if } \left.I \in \mathcal{U}^{n}(K)\right) \text { and } K \in N B^{n} \\ +\infty & \text { otherwise }\end{cases}
$$

11. Set $n:=n+1$ and go to 4

Remark 2.5 In Step 12 of the algorithm described in [4], we defined $u^{n}$ on all the front $F^{n}$, but in the modified version of the GFFM, we define finite values of $u^{n}$ (the useful time) only on the side of the front where we need the values to propagate our computation. Moreover, in this new version, the time at point I $\left(u_{I \rightarrow K}^{n}\right)$ depends on the neighbours $K$ and is defined as the first time the point $I$ becomes useful for $K$.

Remark 2.6 We refer to [4, Subsection 2.3] for a detailed discussion on the complexity and on the implementation of the algorithm. 


\section{Main results}

The physical sequence of time $\left\{t_{n}, n \in \mathbb{N}\right\}$, defined at the step 6 in the algorithm is non-decreasing and we can extract a subsequence $\left\{t_{n_{k}}, k \in \mathbb{N}\right\}$ strictly increasing such that

$$
t_{n_{k}}=t_{n_{k}+1}=\ldots=t_{n_{k+1}-1}<t_{n_{k+1}} .
$$

We denote by $S_{I}^{k}$ the square cell $S_{I}^{k}=\left[x_{I}, x_{I}+\Delta x\left[\times\left[t_{n_{k}}, t_{n_{k+1}}[\right.\right.\right.$ with

$$
\left[x_{I}, x_{I}+\Delta x\left[=\Pi_{\alpha=1}^{N}\left[x_{i_{\alpha}}, x_{i_{\alpha}}+\Delta x[\right.\right.\right.
$$

and by $\varepsilon$ the couple

$$
\varepsilon=(\Delta x, \Delta t)
$$

Let us define the following functions:

$$
\theta^{\varepsilon}(x, t)=\theta_{I}^{n_{k+1}-1} \text { if }(x, t) \in S_{I}^{k} .
$$

Definition 3.1 (Comparable and compared times)

Let $T>0$ and let us consider two velocities $c_{u}$ and $c_{v}$. We denote by $\left(t_{n}\right)_{n}$ and $\left(u_{J \rightarrow K}^{n}\right)_{n}\left(r e s p .\left(s_{m}\right)_{m}\right.$ and $\left.\left(v_{J \rightarrow K}^{m}\right)_{m}\right)$ the sequences of times and of useful times associated to the velocity $c_{u}\left(\right.$ resp. $\left.c_{v}\right)$. We say that $s_{m}$ and $t_{n}$ are comparable if the property $C\left(t_{n}, s_{m}\right)$ holds true:

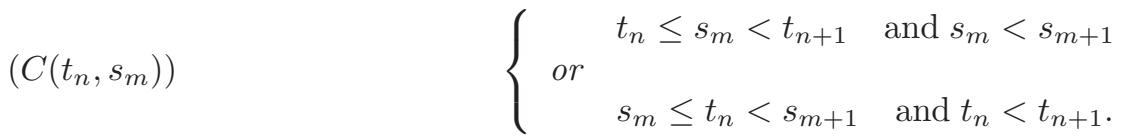

We say that $\theta_{v}^{m}$ and $\theta_{u}^{n}$ are compared and we denote this by $\theta_{v}^{m} \succeq \theta_{u}^{n}$ if for all $J \in \mathbb{Z}^{N}$,

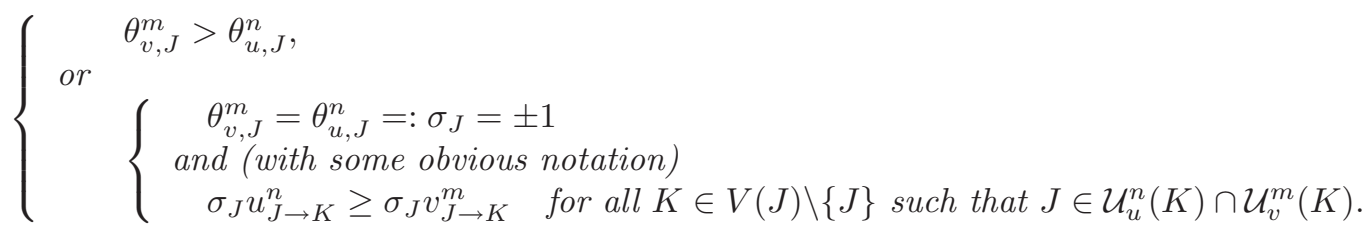

Remark 3.2 The notation $\theta_{v}^{m} \succeq \theta_{u}^{n}$ is abusive. Indeed, this is not an order relation and we also compare the value $u^{n}$ and $v^{m}$ in certain cases.

Remark 3.3 In the definition of $\theta_{v}^{m} \succeq \theta_{u}^{n}$, the fact that the inequality $\sigma_{J} u_{J \rightarrow K}^{n} \geq \sigma_{J} v_{J \rightarrow K}^{m}$ depends on $\sigma_{J}$ can seem curious a priori. The reason is that we search the minimum time and so when $\sigma_{J}=1$, since the point $K$ has to be accepted for $v$ before to be accepted for $u$, we should ask that $u_{J \rightarrow K}^{n} \geq v_{J \rightarrow K}^{m}$. On the contrary, when $\sigma_{J}=-1$, the point $K$ has to be accepted for $u$ before to be accepted for $v$ and so we should ask the reverse inequality.

\section{Theorem 3.4 (Comparison principle for the $\theta^{\varepsilon}$ )}

Let $T>0$. Let two velocities $c_{u}$ and $c_{v}$. Given $\theta_{u, J}^{0}\left(\right.$ resp. $\left.\theta_{v, J}^{0}\right)$ for all $J \in \mathbb{Z}^{N}$ and $u_{J \rightarrow K}^{0}$ for all $J \in \mathcal{U}_{u}^{0}(K)$, $K \in N B_{u}^{0}$ (resp. $v_{J \rightarrow K}^{0}$ for all $J \in \mathcal{U}_{v}^{0}(K), K \in N B_{v}^{0}$ ), we assume that

$$
\begin{gathered}
t_{0}:=\sup _{J \in \mathcal{U}_{u}^{0}(K), K \in N B_{u}^{0}} u_{J \rightarrow K}^{0} \leq T, \\
\left(\text { resp. } s_{0}:=\sup _{J \in \mathcal{U}_{v}^{0}(K), K \in N B_{v}^{0}} v_{J \rightarrow K}^{0} \leq T\right)
\end{gathered}
$$

for a given $T>0$.

We also assume that the two velocities satisfy for all $(x, t) \in \mathbb{R}^{N} \times\left[\min \left(t_{0}, s_{0}\right), T-\Delta t\right]$

$$
\inf _{s \in[t, t+\Delta t]} c_{v}(x, s) \geq \sup _{s \in[t, t+\Delta t]} c_{u}(x, s)
$$

If $C\left(t_{0}, s_{0}\right)$ and $\theta_{v}^{0} \succeq \theta_{u}^{0}$, then

$$
\theta_{v}^{\varepsilon}(x, t) \geq \theta_{u}^{\varepsilon}(x, t)
$$

for all $(x, t) \in \mathbb{R}^{N} \times\left[\max \left(t_{0}, s_{0}\right), T\right]$. 
Remark 3.5 If we choose $u_{J \rightarrow K}^{0}=0$ for all $J \in \mathcal{U}_{u}^{0}(K), K \in N B_{u}^{0}$ and $v_{J \rightarrow K}^{0}=0$ for all $J \in \mathcal{U}_{v}^{0}(K)$, $K \in N B_{v}^{0}$, then the condition $\theta_{v}^{0} \succeq \theta_{u}^{0}$ is equivalent to $\theta_{v, I}^{0} \geq \theta_{u, I}^{0}$ for all $I \in \mathbb{Z}^{N}$.

The basic idea to prove this comparison principle is that when a point $K$ is such that $K \in N B_{u}^{n} \cap N B_{v}^{m}$ with $t_{n}$ and $s_{m}$ comparable (i.e. $C\left(t_{n}, s_{m}\right)$ is true), then all the points $J$ such that $J \in \mathcal{U}_{u}^{n}(K) \cap \mathcal{U}_{v}^{m}(K)$ has become useful for $v$ before to become useful for $u$. Using the definition of the time $u_{J \rightarrow K}^{n}$ and $v_{J \rightarrow K}^{m}$ (Step 10 of the algorithm), we can prove that this implies that $v_{J \rightarrow K}^{m} \leq u_{J \rightarrow K}^{n}$ and we then get that $\tilde{v}_{K}^{m} \leq \tilde{u}_{K}^{n}$ (using the monotonicity of the computation of the candidate time, see Step 4 of the algorithm). It then implies that the point $K$ will be accepted for $v$ before to be accepted for $u$. This preserves the comparison of $\theta_{u}$ and $\theta_{v}$.

We see here that the introduction of a time on each direction is crucial because it allows us to get that $v_{J \rightarrow K}^{m} \leq u_{J \rightarrow K}^{n}$ from the fact that $J$ has become useful for $v$ before to become useful for $u$. This implication is not true if we impose that the time on a point is the same in each direction.

We now give the convergence result for the modified GFMM (presented in Subsection 2.2). To do this, we make the following assumption

(A) The velocity $c \in W^{1, \infty}\left(\mathbb{R}^{N} \times[0, T]\right)$, for some constant $L>0$ we have $\left|c\left(x^{\prime}, t^{\prime}\right)-c(x, t)\right| \leq L\left(\left|x^{\prime}-x\right|+\right.$ $\left.\left|t^{\prime}-t\right|\right)$, and $\Omega_{0}$ is a $C^{2}$ open set, with bounded boundary $\partial \Omega_{0}$.

We also have to define the half-relaxed limits of $\theta^{\varepsilon}$ :

$$
\bar{\theta}^{0}(x, t)=\limsup _{\varepsilon \rightarrow 0, y \rightarrow x, s \rightarrow t} \theta^{\varepsilon}(y, s), \quad \underline{\theta}^{0}(x, t)=\liminf _{\varepsilon \rightarrow 0, y \rightarrow x, s \rightarrow t} \theta^{\varepsilon}(y, s) .
$$

\section{Theorem 3.6 (Convergence Result)}

Under assumption $(A), \bar{\theta}^{0}$ (resp. $\left.\underline{\theta}^{0}\right)$ is a viscosity sub-solution (resp. super-solution) of (1.1). In particular, if (1.1) satisfies a comparison principle, then $\bar{\theta}^{0}=\left(\underline{\theta}^{0}\right)^{*}$ and $\left(\bar{\theta}^{0}\right)_{*}=\underline{\theta}^{0}$ is the unique discontinuous viscosity solution of (1.1).

\section{Preliminary results}

In this section, we give some general properties of the algorithm that will be useful in the proofs.

\section{Proposition 4.1 (General properties of the algorithm)}

We have the following properties:

1. $0 \leq t_{n}-t_{n-1} \leq \Delta t$

2. For all $K \in \mathbb{Z}^{N}$ and $I \in \mathcal{U}^{n}(K)$, we have

$$
u_{I \rightarrow K}^{n} \leq t_{n}
$$

3. $N B^{n} \cap \mathcal{U}^{n}=\emptyset$

4. If $I \in N A^{n}$, then

$$
u_{I \rightarrow K}^{n}= \begin{cases}t_{n} & \text { if } I \in \mathcal{U}^{n}(K) \\ +\infty & \text { otherwise }\end{cases}
$$

5. If $I \in N A^{n}$, then $\tilde{u}_{I}^{n-1} \leq t_{n}$.

6. If $I \in \mathcal{U}^{n-1}(K) \cap \mathcal{U}^{n}(K)$, then

$$
u_{I \rightarrow K}^{n}=u_{I \rightarrow K}^{n-1} .
$$

7. If $I \in \mathcal{U}^{n}(K) \backslash \mathcal{U}^{n-1}(K)$, then

$$
u_{I \rightarrow K}^{n}=t_{n} .
$$

8. If $t_{n}>t_{n-1}$ then $\tilde{t}_{n} \geq t_{n}$.

9. If $t_{n}=t_{n-1}$, then $\tilde{t}_{n} \leq t_{n}$. 


\section{Proof of Proposition 4.1}

1. This is a straightforward consequence of Point 6 of the algorithm.

2. This is a straightforward consequence of Point 10 of the algorithm.

3. By contradiction, assume that there exists $I$ and $K \in V(I) \backslash\{I\}$ such that $I \in N B^{n} \cap \mathcal{U}^{n}(K)$. The fact that $I \in N B^{n}$ implies that

$$
\theta_{I}^{n} \hat{c}_{I}^{n}<0
$$

The fact that $I \in \mathcal{U}^{n}(K)$ implies that

$$
\theta_{I}^{n}=-\theta_{K}^{n} \quad \text { and } \quad \theta_{I}^{n} \hat{c}_{K}^{n}>0 .
$$

Combining (4.1) and (4.2), we get

$$
\hat{c}_{I}^{n} \hat{c}_{K}^{n}<0
$$

which contradicts the definition of $\hat{c}$.

4. By point 10 of the algorithm, we just have to prove that if $I \in N A^{n}$, then $\min \left(u_{I \rightarrow K}^{n-1}, t_{n}\right)=t_{n}$. But if $I \in N A^{n}$, then $I \in N B^{n-1}$ and so, by Property 3, we get that $I \notin \mathcal{U}^{n-1}(K)$. This implies that $u_{I \rightarrow K}^{n-1}=+\infty$.

5. By contradiction, assume that $\tilde{u}_{I}^{n-1}>t_{n}$. Since $I \in N A^{n}$, we get that

$$
\tilde{u}_{I}^{n-1}=\tilde{t}_{n}>t_{n} .
$$

By Step 6 of the algorithm, we deduce that $t_{n}=t_{n-1}+\Delta t<\tilde{t}_{n}$ and so by Step 7 , no point are accepted. Contradiction.

6. If $I \in \mathcal{U}^{n-1}(K) \cap \mathcal{U}^{n}(K)$, then by Step 10 of the algorithm, we have

$$
u_{I \rightarrow K}^{n}=\min \left(u_{I \rightarrow K}^{n-1}, t_{n}\right)=u_{I \rightarrow K}^{n-1}
$$

where we have used Property 2 for the last equality.

7. If $I \in \mathcal{U}^{n}(K) \backslash \mathcal{U}^{n-1}(K)$, then by Step 10 of the algorithm, we have

$$
u_{I \rightarrow K}^{n}=\min \left(u_{I \rightarrow K}^{n-1}, t_{n}\right)=t_{n}
$$

where we have used that $u_{I \rightarrow K}^{n-1}=+\infty$ since $I \notin \mathcal{U}^{n-1}(K)$.

8. If $t_{n}>t_{n-1}$, then by Step 6 of the algorithm, we get

$$
t_{n}=\min \left(\tilde{t}_{n}, t_{n-1}+\Delta t\right) \leq \tilde{t}_{n} .
$$

9. If $t_{n}=t_{n-1}$, then by Step 6 of the algorithm, we get

$$
t_{n-1} \geq \min \left(\tilde{t}_{n}, t_{n-1}+\Delta t\right)
$$

and so $t_{n}=t_{n-1} \geq \tilde{t}_{n}$.

Proposition 4.2 (Monotonicity of $c \rightarrow \hat{c}$ )

The application $c \mapsto \hat{c}$ is monotone. More precisely, if $c_{v} \geq c_{u}$ then $\hat{c}_{v} \geq \hat{c}_{u}$.

Proof of Proposition 4.2 Let $c_{v} \geq c_{u}$. We will prove that for all $I \in \mathbb{Z}^{N}$, we have

$$
\hat{c}_{v, I} \geq \hat{c}_{u, I} .
$$

The proof is distinguished in four cases: 
Case 1: $\hat{c}_{v, I}=c_{v, I}$ and $\hat{c}_{u, I}=c_{u, I}$.

In this case, we have

$$
\hat{c}_{v, I}=c_{v, I} \geq c_{u, I}=c_{u, I}
$$

and (4.3) is true.

Case 2: $\hat{c}_{v, I} \neq c_{v, I}$ and $\hat{c}_{u, I} \neq c_{u, I}$.

In this case, we have $\hat{c}_{v, I}=0=\hat{c}_{u, I}$ and so (4.3) is true.

Case 3: $\hat{c}_{v, I}=c_{v, I}$ and $\hat{c}_{u, I} \neq c_{u, I}$.

This implies in particular that $\hat{c}_{u, I}=0$. By contradiction, let us assume that

$$
\hat{c}_{v, I}=c_{v, I}<0=\hat{c}_{u, I}
$$

Since $c_{v} \geq c_{u}$, this implies in particular that $c_{u, I}<0$. Since $\hat{c}_{u, I} \neq c_{u, I}$, we also deduce that there exists $J \in V(I)$ such that $c_{u, J}>0$ and $\left|c_{u, I}\right| \leq\left|c_{u, J}\right|$. This implies that

$$
\left|c_{v, I}\right| \leq\left|c_{u, I}\right| \leq\left|c_{u, J}\right|=c_{u, J} \leq c_{v, J}
$$

and so $\hat{c}_{v, I}=0$. This contradicts (4.4) and proves (4.3).

Case 4: $\hat{c}_{v, I} \neq c_{v, I}$ and $\hat{c}_{u, I}=c_{u, I}$.

This case can be treated in the same way as Case 3 .

\section{Proposition 4.3 (Symmetry of the algorithm)}

Let us consider two velocities $c_{u}$ and $c_{v}$. We denote by $\left(t_{n}^{u}\right)_{n},\left(u_{J \rightarrow K}^{n}\right)_{n},\left(\theta_{u}^{n}\right)_{n}\left(\right.$ resp. $\left.\left(s_{m}^{v}\right)_{m},\left(v_{J \rightarrow K}^{m}\right)_{m},\left(\theta_{v}^{m}\right)_{m}\right)$ the sequences of times, of useful times and of field associated to the velocity $c_{u}$ (resp $c_{v}$ ) and with the initial condition $\theta_{u}^{0}\left(\right.$ resp. $\left.\theta_{v}^{0}\right)$.

We also define $\left(s_{m}^{\bar{u}}\right)_{m},\left(\bar{u}_{J \rightarrow K}^{m}\right)_{m},\left(\theta_{\bar{u}}^{m}\right)_{m}$ (resp. $\left.\left(t_{n}^{\bar{v}}\right)_{n},\left(\bar{v}_{J \rightarrow K}^{n}\right)_{n},\left(\theta_{\bar{v}}^{n}\right)_{n}\right)$ the sequences of times, of useful times and of field associated to the velocity $c_{\bar{u}}=-c_{v}\left(\right.$ resp $\left.c_{\bar{v}}=-c_{u}\right)$ and with the initial condition $\theta_{\bar{u}}^{0}=-\theta_{v}^{0}$ $\left(\right.$ resp. $\left.\theta_{\bar{v}}^{0}=-\theta_{u}^{0}\right)$.

Then, we have the following equivalence:

$$
\theta_{v}^{m} \succeq \theta_{u}^{n} \Longleftrightarrow \theta_{\bar{v}}^{n} \succeq \theta_{\bar{u}}^{m}
$$

\section{Proof of Proposition 4.3}

Following [4, Lemma 4.1], we have

$$
\theta_{\bar{u}}^{m}=-\theta_{v}^{m}, \quad \theta_{\bar{v}}^{n}=-\theta_{u}^{n}, \quad s_{m}^{\bar{u}}=s_{m}^{v}, \quad t_{n}^{\bar{v}}=t_{n}^{u}, \quad \bar{u}_{J \rightarrow K}^{m}=v_{J \rightarrow K}^{m}, \quad \bar{v}_{J \rightarrow K}^{n}=u_{J \rightarrow K}^{n}
$$

Let us assume that $\theta_{v}^{m} \succeq \theta_{u}^{n}$ and let us prove that $\theta_{\bar{u}}^{m} \succeq \theta_{\bar{v}}^{n}$ (the other implication being similar).

Case 1: $\theta_{v}^{m}>\theta_{u}^{n}$

Then

$$
\theta_{\bar{u}}^{m}=-\theta_{v}^{m}<-\theta_{u}^{n}=\theta_{\bar{v}}^{n}
$$

Case 2: $\theta_{v}^{m}=\theta_{u}^{n}=\sigma$

This implies that

$$
\theta_{\bar{u}}^{m}=\theta_{\bar{v}}^{n}=\bar{\sigma}=-\sigma .
$$

Let $J \in \mathcal{U}_{\bar{u}}^{m}(K) \cap \mathcal{U}_{\bar{v}}^{n}(K)$. This implies in particular that $J \in \mathcal{U}_{v}^{m}(K) \cap \mathcal{U}_{u}^{n}(K)$. Then, we have

$$
\bar{\sigma} \bar{v}_{J \rightarrow K}^{n}=-\sigma u_{J \rightarrow K}^{n} \leq-\sigma v_{J \rightarrow K}^{m}=\bar{\sigma} \bar{u}_{J \rightarrow K}^{m}
$$

where we have used that $\theta_{v}^{m} \succeq \theta_{u}^{n}$.

This implies that $\theta_{\bar{v}}^{n} \succeq \theta_{\bar{u}}^{m}$. 


\section{Comparison principle}

\subsection{Comparison principle for $\theta^{\varepsilon}$}

This subsection is devoted to the proof of Theorem 3.4.

To prove this theorem, we need the following comparison result for the $t_{n}$ time, whose proof is given in Subsection 5.3.

\section{Theorem 5.1 (Comparison principle for the $t_{n}$ times)}

Under the assumptions of Theorem 3.4, we denote by $\left(t_{n}\right)_{n}$ and $\left(u_{J \rightarrow K}^{n}\right)_{n}$ (resp. $\left(s_{m}\right)_{m}$ and $\left.\left(v_{J \rightarrow K}^{m}\right)_{m}\right)$ the sequences of times and of useful times associated to the velocity $c_{u}$ (resp. $c_{v}$ ). Then $\theta_{v}^{m} \succeq \theta_{u}^{n}$ for every times satisfying $\left(C\left(t_{n}, s_{m}\right)\right)$ and $t_{n}, s_{m} \leq T$.

\section{Proof of Theorem 3.4}

Let us define

$$
t^{*}=\inf \left\{t \geq \max \left(t_{0}, s_{0}\right) \quad \text { s.t. } \quad \theta_{v}^{\varepsilon}(x, t)<\theta_{u}^{\varepsilon}(x, t) \text { for some } x \in \mathbb{R}^{N}\right\}
$$

and

$$
x^{*} \quad \text { s.t. } \quad \theta_{v}^{\varepsilon}\left(x^{*}, t^{*}\right)<\theta_{u}^{\varepsilon}\left(x^{*}, t^{*}\right) .
$$

Let $m, n$ and $J$ s.t.

$$
\left\{\begin{array}{l}
s_{m} \leq t^{*}<s_{m+1} \\
t_{n} \leq t^{*}<t_{n+1} \\
x^{*} \in\left[x_{J}, x_{J}+\Delta x\right)
\end{array}\right.
$$

Then $\theta_{v}^{\varepsilon}\left(x^{*}, t\right)=\theta_{v, J}^{m}$ for $t \in\left[s_{m}, s_{m+1}\right)$ and $\theta_{u}^{\varepsilon}\left(x^{*}, t\right)=\theta_{u, J}^{n}$ for $t \in\left[t_{n}, t_{n+1}\right)$. Therefore $t^{*}=s_{m}$ or $t^{*}=t_{n}$ and we can easily check that $C\left(t_{n}, s_{m}\right)$ is true. This implies by Theorem 5.1 that $\theta_{v}^{m} \succeq \theta_{u}^{n}$ and then

$$
\theta_{v, J}^{m} \geq \theta_{u, J}^{n}
$$

which is a contradiction.

\subsection{Counter-example for GFMM of [4]}

In this subsection, we give a counter-example in dimension one for the comparison principle for the GFMM of [4], which holds for all $\Delta x$ and $\Delta t$ small enough. We recall that in the GFMM of [4], we affect, to $u^{n}$ (for points that are not accepted) the time when the point enter in the front while in the GFMM of Subsection 2.2 , we affect the time when the point becomes useful.

The velocity for $c_{u}$ and $c_{v}$ are given by

$$
c_{u}(x, t)=\left\{\begin{array}{ll}
\max \left(0, \frac{4}{3} x\right) & \text { if } t \leq 1 \\
\min (0,-2 x) & \text { if } t \geq \frac{17}{16}
\end{array}, \quad c_{v}(x, t)= \begin{cases}\max (0, x) & \text { if } t \leq 1 \\
\min (0,-3 x) & \text { if } t \geq \frac{17}{16}\end{cases}\right.
$$

and $c_{u}, c_{v} \in \operatorname{Lip}\left(\mathbb{R}^{N} \times[0, T]\right)$. The initial conditions are given by

$$
\theta_{u, i}^{0}=\theta_{v, i}^{0}=\left\{\begin{array}{lll}
1 & \text { if } \quad i \leq 0 \\
-1 & \text { if } \quad i \geq 1
\end{array}\right.
$$

We begin to compute the evolution for $u$. The result are presented in Figure 2 on the left.

A simple computation gives that the point $i=1$ will be accepted at time $t=\frac{3}{4}$ and we affect the value $u_{1}=u_{2}=\frac{3}{4}$. The point $i=2$ will not be accepted before the velocity changes sign because $\tilde{u}_{2}=\frac{9}{8}>\frac{17}{16}$. When the velocity changes sign, the point $i=1$ will be accepted again for $\Delta t$ small enough, with a time

$$
u_{1}=u_{2}+\frac{\Delta x}{2 \Delta x}=\frac{5}{4}>\frac{17}{16}
$$

We now compute the evolution for $v$. The result are presented in Figure 2 on the right. As before, the point $i=1$ will be accepted at time $s=1$ and the point $i=2$ will not be accepted before the velocity 
changes sign. Moreover, $v_{2}=1$. When the velocity changes sign, the point $i=1$ will be again accepted with a time

$$
v_{1}=v_{2}+\frac{\Delta x}{3 \Delta x}=\frac{4}{3}>\frac{5}{4} .
$$

As we see, during the time interval $[5 / 4,4 / 3]$, we have

$$
\theta_{u}^{\varepsilon}(\Delta x, t)=-1 \quad \text { and } \quad \theta_{v}^{\varepsilon}(\Delta x, t)=1
$$

which contradicts the comparison principle.
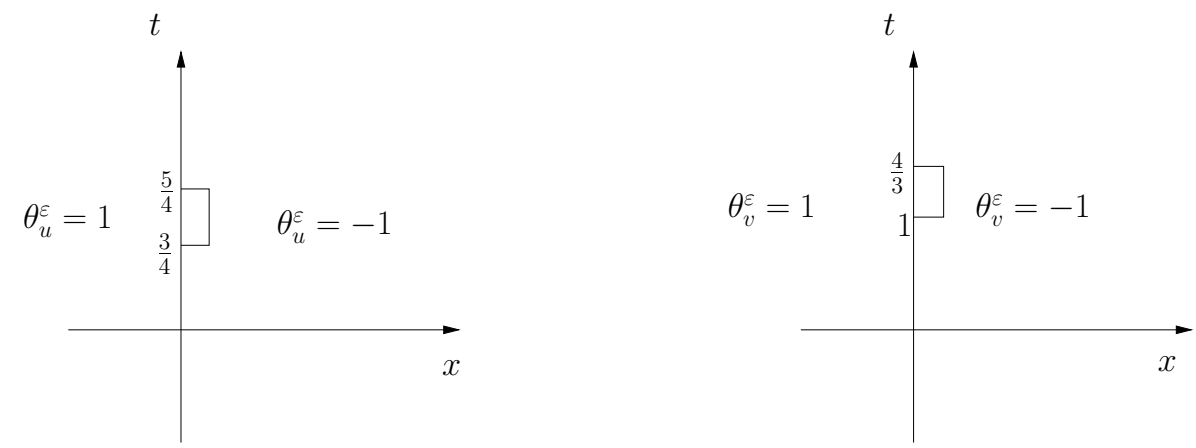

Figure 2: Counter-example for the comparison principle.

Remark 5.2 In this counter-example, we see that the contradiction comes from the value we put for the node $i=2$ which is the time when it enters in the front. This is the reason why, in our new GFFM (see Subsection 2.2), we put the time when the point become useful. Since the velocity are ordered, we then have $u_{2} \leq v_{2}$ and so the evolution preserves the inclusion (see Theorem 3.4).

\subsection{Comparison principle for the $t_{n}$ times}

This subsection is devoted to the proof of Theorem 5.1. To perform the proof, we will need the following results

Lemma 5.3 (Two jumps, two arrivals)

Assume that $\theta_{v}^{m-1} \succeq \theta_{u}^{n-1}$. Then, if $s_{m-1} \leq t_{n}<s_{m}$ (resp. $t_{n-1} \leq s_{m}<t_{n}$ ) and $t_{n}, s_{m}<T$, then we have $\theta_{v}^{m-1} \succeq \theta_{u}^{n}\left(\right.$ resp. $\left.\theta_{v}^{m} \succeq \theta_{u}^{n-1}\right)$.

\section{Lemma 5.4 (Two jumps, one arrival)}

Assume that $\theta_{v}^{m-1} \succeq \theta_{u}^{n-1}, t_{n-1}<t_{n}$ and $s_{m-1}<s_{m}$. If $s_{m}=t_{n} \leq T$, then $\theta_{v}^{m} \succeq \theta_{u}^{n}$.

\section{Proposition 5.5 (Stationary case with the same arrival)}

Let us assume that $t_{n-p-1}<t_{n-p}=\ldots=t_{n}<t_{n+1}$ and $s_{m-q-1}<s_{m-q}=\ldots=s_{m}<s_{m+1}$ with $p, q \geq 0$ and $t_{n}=s_{m} \leq T$. If $\theta_{v}^{m-q} \succeq \theta_{u}^{n-p}$, then $\theta_{v}^{m} \succeq \theta_{u}^{n}$.

The proofs of Lemma 5.3 and 5.4 are given at the end of this subsection while the proof of Proposition 5.5 is given in Section 6 .

\section{Proof of Theorem 5.1}

By contradiction, let us define

$$
s^{*}=\inf \left\{\min \left(t_{n}, s_{m}\right) \text { such that } \theta_{v}^{m} \nsucceq \theta_{u}^{n}, t_{n}, s_{m} \leq T \text { and } C\left(t_{n}, s_{m}\right) \text { is true }\right\}
$$

Up to a symmetry (see Proposition 4.3), we can assume that $s^{*}=s_{m^{*}}$ for a certain index $m^{*}$. Then let us define

$$
n^{*}=\inf \left\{n \geq 0 \text { such that } \theta_{v}^{m^{*}} \nsucceq \theta_{u}^{n}, t_{n} \leq T \text { and } C\left(t_{n}, s_{m^{*}}\right) \text { is true }\right\}
$$

Therefore $\left(t_{n^{*}}, s_{m^{*}}\right)$ is the minimal couple such that $\theta_{v}^{m^{*}} \nsucceq \theta_{u}^{n^{*}}, s_{m^{*}} \leq t_{n^{*}}<s_{m^{*}+1}$ and $t_{n^{*}}<t_{n^{*}+1}$.

To simplify the notation, let us denote $n^{*}$ by $n$ and $m^{*}$ by $m$. The proof is decomposed into two cases: 
Case 1: $s_{m}<t_{n}$

We define $p \geq 0$ such that

$$
t_{n-p-1}<t_{n-p}=\ldots=t_{n}<t_{n+1} .
$$

Step $1: \theta_{v}^{m} \succeq \theta_{u}^{n-p-1}$

To prove that $\theta_{v}^{m} \succeq \theta_{u}^{n-p-1}$ is true, it suffices to show that $C\left(t_{n-p-1}, s_{m}\right)$ is satisfied (because $(n, m)$ is the minimal couple such that $\left.\theta_{v}^{m} \nsucceq \theta_{u}^{n}\right)$. We recall that

$$
s_{m}<t_{n-p}=\ldots=t_{n}<s_{m+1}, \quad \text { and } \quad t_{n-p+1}<t_{n-p} .
$$

If $s_{m} \leq t_{n-p-1}$, then $s_{m} \leq t_{n-p-1}<s_{m+1}$ and $t_{n-p-1}<t_{n-p}$, while if $t_{n-p-1}<s_{m}$, then $t_{n-p-1}<$ $s_{m}<t_{n-p}$ and $s_{m}<s_{m+1}$.

So, we always have that property $C\left(t_{n-p-1}, s_{m}\right)$ is satisfied, which implies that $\theta_{v}^{m} \succeq \theta_{u}^{n-p-1}$.

\section{Step 2: Contradiction.}

Using Lemma 5.3 successively, we deduce that $\theta_{v}^{m} \succeq \theta_{u}^{n-p}, \ldots, \theta_{v}^{m} \succeq \theta_{u}^{n}$. Contradiction.

Case 2: $s_{m}=t_{n}$

Let us define $p$ and $q$ such that

$$
t_{n-p-1}<t_{n-p}=\ldots=t_{n}<t_{n+1} \quad \text { and } \quad s_{m-q-1}<s_{m-q}=\ldots=s_{m}<s_{m+1} .
$$

We then have $t_{n-p-1} \leq s_{m-q-1}<s_{m-q}=t_{n-p}$ or $s_{m-q-1} \leq t_{n-p-1}<t_{n-p}=s_{m-q}$ and so $C\left(t_{n-p-1}, s_{m-q-1}\right)$ is satisfied. Therefore, this implies (by definition of $\left.(n, m)\right)$ that $\theta_{v}^{m-q-1} \succeq \theta_{u}^{n-p-1}$. Using Lemma 5.4, we deduce that $\theta_{v}^{m-q} \succeq \theta_{u}^{n-p}$. Using Proposition 5.5, we then deduce that $\theta_{v}^{m} \succeq \theta_{u}^{n}$. Contradiction.

Before to give the proofs of Lemma 5.3 and 5.4, we need some preliminary results (Lemma 5.6 and 5.7).

The following lemma claims that if we compare $\theta_{u}^{n-1}$ and $\theta_{v}^{m-1}$ (in the sense $\theta_{v}^{m-1} \succeq \theta_{u}^{n-1}$ ), then we can compare the candidate time $\tilde{u}^{n-1}$ and $\tilde{v}^{m-1}$ on the Narrow Band. This essentially comes from our definition of $\theta_{v}^{m-1} \succeq \theta_{u}^{n-1}$ in which we compare not only the $\theta$ (and so the point that will play a role in the computation of the candidate time) but also the time on the useful points. The result then comes from the monotonicity of the computation of the candidate time (see Step 4 of the algorithm).

\section{Lemma 5.6 (Comparison of the candidate time $\tilde{u}$ )}

Assume that $\theta_{v}^{m-1} \succeq \theta_{u}^{n-1},\left|t_{n-1}-s_{m-1}\right| \leq \Delta t$, with $t_{n-1}, s_{m-1} \leq T$. If

$$
\begin{gathered}
\theta_{v, I}^{m-1}=\theta_{u, I}^{n-1}=-1 \quad \text { and } \quad I \in N B_{u,-}^{n-1} \\
\left(\text { resp. } \quad \theta_{v, I}^{m-1}=\theta_{u, I}^{n-1}=1 \quad \text { and } \quad I \in N B_{v,+}^{m-1}\right),
\end{gathered}
$$

then

$$
\begin{gathered}
I \in N B_{v,-}^{m-1} \text { and } \tilde{u}_{I}^{n-1} \geq \tilde{v}_{I}^{m-1} \\
\text { (resp. } \left.I \in N B_{u,+}^{n-1} \quad \text { and } \quad \tilde{u}_{I}^{n-1} \leq \tilde{v}_{I}^{m-1}\right) .
\end{gathered}
$$

Proof of Lemma 5.6

We do the proof in the case $\theta_{v, I}^{m-1}=\theta_{u, I}^{n-1}=-1$ and $I \in N B_{u,-}^{n-1}$ (the other case is similar). Since $I \in N B_{u,-}^{n-1}$, we deduce that

$$
\left\{\begin{array}{l}
\exists J \in V(I) \backslash\{I\} \text { s.t. } \theta_{u, J}^{n-1}=1 \\
\hat{c}_{u, I}^{n-1}>0
\end{array}\right.
$$

Step 1: $I \in N B_{v,-}^{m-1}$

Since $\theta_{v}^{m-1} \succeq \theta_{u}^{n-1}$, we deduce that $\theta_{v, J}^{m-1} \geq \theta_{u, J}^{n-1}=1$ and so $\theta_{v, J}^{m-1}=1$. This implies that

$$
I \in F_{v,-}^{m-1} .
$$

Moreover $\left|t_{n-1}-s_{m-1}\right| \leq \Delta t$ and so

$$
c_{v}\left(\cdot, s_{m-1}\right) \geq c_{u}\left(\cdot, t_{n-1}\right) .
$$


Using Proposition 4.2, yields

$$
\hat{c}_{v, I}^{m-1} \geq \hat{c}_{u, I}^{n-1}>0
$$

where the last inequality comes from (5.1).

Combining (5.2) and (5.3), we deduce that

$$
I \in N B_{v,-}^{m-1}
$$

Step 2: Ordering $u_{\cdot \rightarrow I}^{n-1} \geq v_{\cdot \rightarrow I}^{m-1}$

Let $J \in V(I) \backslash\{I\}$.

Case 1: $\theta_{v, J}^{m-1}=-1$

Since $\theta_{v}^{m-1} \succeq \theta_{u}^{n-1}$, we get that $\theta_{v, J}^{m-1} \geq \theta_{u, J}^{n-1}$ and then $\theta_{u, J}^{n-1}=-1$. Therefore

$$
u_{J \rightarrow I}^{n-1}=+\infty=v_{J \rightarrow I}^{m-1}
$$

Case 2: $\theta_{v, J}^{m-1}=+1$

Assume first that $\theta_{u, J}^{n-1}=+1$ which implies that $J \in \mathcal{U}_{u}^{n-1}(I) \cap \mathcal{U}_{v}^{m-1}(I)$. Then assumption $\theta_{v}^{m-1} \succeq \theta_{u}^{n-1}$ implies that $u_{J \rightarrow I}^{n-1} \geq v_{J \rightarrow I}^{m-1}$ with $\sigma_{J}=+$.

In the case $\theta_{u, J}^{n-1}=-1$, we get that $u_{J \rightarrow I}^{n-1}=+\infty$ which again implies $u_{J \rightarrow I}^{n-1} \geq v_{J \rightarrow I}^{m-1}$.

We therefore deduce that in the general case, we always have

$$
u_{J \rightarrow I}^{n-1} \geq v_{J \rightarrow I}^{m-1} \quad \text { for any } \quad J \in V(I) \backslash\{I\} .
$$

Step 3: $\tilde{u}_{I}^{n-1} \geq \tilde{v}_{I}^{m-1}$

We know that

$$
\sum_{k=1}^{N}\left(\max \left(0, \tilde{u}_{I}^{n-1}-u_{I^{k, \pm} \rightarrow I}^{n-1}\right)\right)^{2}=\frac{(\Delta x)^{2}}{\left|\hat{c}_{u, I}^{n-1}\right|^{2}}
$$

and

$$
\sum_{k=1}^{N}\left(\max \left(0, \tilde{v}_{I}^{m-1}-v_{I^{k, \pm} \rightarrow I}^{m-1}\right)\right)^{2}=\frac{(\Delta x)^{2}}{\left|\hat{c}_{v, I}^{m-1}\right|^{2}}
$$

with $\hat{c}_{v, I}^{m-1} \geq \hat{c}_{u, I}^{n-1}>0$ (see (5.3)), which implies, using also Step 2 that

$$
\tilde{u}_{I}^{n-1} \geq \tilde{v}_{I}^{m-1}
$$

and ends the proof of the Lemma.

The following lemma claims that a point $J$ with $\theta_{v, J}^{m}=\theta_{u, J}^{n}=-1$ is useful for $u$ before to be useful for $J$. This essentially comes from the fact that the velocity are ordered.

\section{Lemma 5.7 (Property of useful points)}

Assume that $J \in \mathcal{U}_{v}^{m}(K) \cap \mathcal{U}_{u}^{n}(K), \theta_{v, J}^{m}=\theta_{u, J}^{n}=\theta_{u, J}^{n-1}=-1$ and $\left|t_{n-1}-s_{m}\right| \leq \Delta t$ with $t_{n}, s_{m} \leq T$.

Then $J \in \mathcal{U}_{u}^{n-1}(K)$.

\section{Proof of Lemma 5.7}

By contradiction, assume that $J \notin \mathcal{U}_{u}^{n-1}(K)$. Since $J \in \mathcal{U}_{v}^{m}(K)$ and $\theta_{v, J}^{m}=-1$, we deduce that

$$
\theta_{v, K}^{m}=1 \quad \text { and } \quad \hat{c}_{v, K}^{m}<0 \text {. }
$$

In the same way, we also have

$$
\theta_{u, K}^{n}=1 \quad \text { and } \quad \hat{c}_{u, K}^{n}<0
$$

Now because $\left|t_{n-1}-s_{m}\right| \leq \Delta t$, we deduce that

$$
\hat{c}_{u, K}^{n-1} \leq \hat{c}_{v, K}^{m}<0 .
$$

Using that $\theta_{u, J}^{n-1}=-1, \hat{c}_{u, K}^{n-1}<0$ and $J \notin \mathcal{U}_{u}^{n-1}(K)$, we deduce that

$$
\theta_{u, J}^{n-1}=\theta_{u, K}^{n-1}=-1
$$

But we can not pass from $\theta_{u, K}^{n-1}=-1$ to $\theta_{u, K}^{n}=+1$ with a negative velocity $\hat{c}_{u, K}^{n-1}<0$. Contradiction. 


\section{Proof of Lemma 5.3}

We make the proof in the case $s_{m}>t_{n}$, the other case being equivalent by symmetry (see Proposition 4.3). By contradiction, assume that $\theta_{v}^{m-1} \nsucceq \theta_{u}^{n}$. Then, there exists $J$ such that

$$
\left\{\begin{array}{l}
\theta_{v, J}^{m-1}<\theta_{u, J}^{n} \\
\text { or } \\
\theta_{v, J}^{m-1}=\theta_{u, J}^{n}=\sigma_{J} \text { and } \exists K: J \in \mathcal{U}_{v}^{m-1}(K) \cap \mathcal{U}_{u}^{n}(K) \text { and } \sigma_{J} u_{J \rightarrow K}^{n}<\sigma_{J} v_{J \rightarrow K}^{m-1}
\end{array}\right.
$$

The proof is decomposed in two cases: in the first one, the value of $\theta_{u}^{n}$ changes while it do not change in the second one.

Case 1: $J \in N A_{u}^{n}$

There is only two non trivial sub-cases (but we treat all the cases below). The first one (Sub-case 1.1) is when the two $\theta$ were different and are now the same. In this case, we will contradicts the second line of (5.5) (on the time). This is rather easy because we know the value of $u_{J \rightarrow K}^{n}$ (which is in fact $t_{n}$ since $J \in N A_{u}^{n}$ ). The second non trivial sub-case (Sub-case 1.2.2) is when the $\theta$ were the same and are now order in the bad sense (first line of (5.5)). In this case, we will prove that $J$ should have been accepted for $v$ before to be accepted for $u$.

Sub-case 1.1: $\theta_{v, J}^{m-1}>\theta_{u, J}^{n-1}$

Then $\theta_{v, J}^{m-1}=1$ and $\theta_{u, J}^{n-1}=-1$. Since $J \in N A_{u}^{n}$, we deduce that $\theta_{u, J}^{n}=1=\theta_{v, J}^{m-1}=\sigma_{J}$. We then have

$$
v_{J \rightarrow K}^{m-1} \leq s_{m-1} \leq t_{n}=u_{J \rightarrow K}^{n}
$$

where we have used the fact that $J \in \mathcal{U}_{v}^{m-1}(K)$ and Proposition 4.1 Point 2 for the first inequality and the fact that $J \in N A_{u}^{n} \cap \mathcal{U}_{u}^{n}(K)$ joint to Proposition 4.1 Point 4 for the last equality. This contradicts (5.5).

Sub-case 1.2: $\theta_{v, J}^{m-1}=\theta_{u, J}^{n-1}$

Sub-case 1.2.1: $\theta_{v, J}^{m-1}=\theta_{u, J}^{n-1}=1$

Since $J \in N A_{u}^{n}$, we deduce that $\theta_{u, J}^{n}=-1$. This contradicts (5.5).

Sub-case 1.2.2: $\theta_{v, J}^{m-1}=\theta_{u, J}^{n-1}=-1$

Step 1: $\tilde{u}_{J}^{n-1} \geq \tilde{v}_{J}^{m-1}$ and $J \in N B_{v,-}^{m-1}$

We want to apply Lemma 5.6. We recall that $\theta_{v}^{m-1} \succeq \theta_{u}^{n-1}$ and $\theta_{v, J}^{m-1}=\theta_{u, J}^{n-1}=-1$. Since $J \in N A_{u}^{n}$, we also deduce that $J \in N B_{u,-}^{n-1}$. Moreover $\left|t_{n-1}-s_{m-1}\right| \leq \Delta t$ (because $s_{m}-s_{m-1} \leq \Delta t$ and $t_{n}-t_{n-1} \leq \Delta t$ ). We can then apply Lemma 5.6 to get

$$
\tilde{u}_{J}^{n-1} \geq \tilde{v}_{J}^{m-1} \text { and } \quad J \in N B_{v,-}^{m-1}
$$

Step 2: $\tilde{v}_{J}^{m-1} \geq s_{m}$

Recall that $s_{m}=\max \left(s_{m-1}, \min \left(s_{m-1}+\Delta t, \tilde{s}_{m}\right)\right)$. But $s_{m}>s_{m-1}$ and so

$$
s_{m}=\min \left(s_{m-1}+\Delta t, \tilde{s}_{m}\right) \leq \tilde{s}_{m} .
$$

Since, by definition of $\tilde{s}_{m}$, we have $\tilde{s}_{m} \leq \tilde{v}_{J}^{m-1}$ with $J \in N B_{v,-}^{m-1}$ (Step 1), we deduce that

$$
\tilde{v}_{J}^{m-1} \geq s_{m}
$$

\section{Step 3: Contradiction}

By Step 1 and 2, we get

$$
\tilde{u}_{J}^{n-1} \geq \tilde{v}_{J}^{m-1} \geq s_{m}>t_{n}
$$

and then

$$
\tilde{u}_{J}^{n-1}>t_{n}
$$

This is impossible because $J \in N A_{u}^{n}$ (see Proposition 4.1 Point 5).

Sub-case 1.3: $\theta_{v, J}^{m-1}<\theta_{u, J}^{n-1}$

This is impossible since $\theta_{v}^{m-1} \succeq \theta_{u}^{n-1}$. 
Case 2: $J \notin N A_{u}^{n}$

In this case, the $\theta$ do not change and we essentially have to contradict the second line of (5.5). There is essentially two cases. The first one is when the value of $u_{J \rightarrow K}^{n}$ has changed and we will use that $u_{J \rightarrow K}^{n}=t_{n}$. The second one is when $u_{J \rightarrow K}^{n}=u_{J \rightarrow K}^{n-1}$ and we will use assumption $\theta_{v, J}^{m-1} \succeq \theta_{u, J}^{n-1}$ to get the contradiction.

Sub-case 2.1: $\theta_{v, J}^{m-1}<\theta_{u, J}^{n}$

Since $J \notin N A_{u}^{n}$, we have $\theta_{u, J}^{n-1}=\theta_{u, J}^{n}$ and so $\theta_{v, J}^{m-1}<\theta_{u, J}^{n-1}$. Contradiction with $\theta_{v}^{m-1} \succeq \theta_{u}^{n-1}$.

Sub-case 2.2: $\theta_{v, J}^{m-1}=\theta_{u, J}^{n}$

Since $J \notin N A_{u}^{n}$, we have

$$
\theta_{u, J}^{n-1}=\theta_{u, J}^{n}=\theta_{v, J}^{m-1}=\sigma_{J}= \pm 1 .
$$

From (5.5), there exists $K$ such that

$$
J \in \mathcal{U}_{v}^{m-1}(K) \cap \mathcal{U}_{u}^{n}(K) \text { and } \sigma_{J} u_{J \rightarrow K}^{n}<\sigma_{J} v_{J \rightarrow K}^{m-1}
$$

We then distinguish several sub-cases:

Sub-case 2.2.1: $J \in \mathcal{U}_{u}^{n-1}(K)$

From (5.7), we have $J \in \mathcal{U}_{v}^{m-1}(K) \cap \mathcal{U}_{u}^{n-1}(K)$. Now the fact that $\theta_{v}^{m-1} \succeq \theta_{u}^{n-1}$ and (5.6) imply that

$$
\sigma_{J} u_{J \rightarrow K}^{n-1} \geq \sigma_{J} v_{J \rightarrow K}^{m-1}
$$

Now $J \in \mathcal{U}_{u}^{n-1}(K) \cap \mathcal{U}_{u}^{n}(K)$ imply, by Proposition 4.1 Point 6, that $u_{J \rightarrow K}^{n}=u_{J \rightarrow K}^{n-1}$. Therefore

$$
\sigma_{J} u_{J \rightarrow K}^{n} \geq \sigma_{J} v_{J \rightarrow K}^{m-1}
$$

which contradicts (5.7).

Sub-case 2.2.2: $J \notin \mathcal{U}_{u}^{n-1}(K)$ and $\sigma_{J}=+1$

Using the fact that $J \in \mathcal{U}_{u}^{n}(K) \backslash \mathcal{U}_{u}^{n-1}(K)$ joint to Proposition 4.1 Point 7, we get that $u_{J \rightarrow K}^{n}=t_{n}$. This implies that

$$
u_{J \rightarrow K}^{n}=t_{n} \geq s_{m-1} \geq v_{J \rightarrow K}^{m-1}
$$

where we have used the fact that $J \in \mathcal{U}_{v}^{m-1}(K)$ joint to Proposition 4.1 Point 2 for the last inequality. Contradiction with (5.7).

Sub-case 2.2.3: $J \notin \mathcal{U}_{u}^{n-1}(K)$ and $\sigma_{J}=-1$

In this case, we have $J \in \mathcal{U}_{v}^{m-1}(K) \cap \mathcal{U}_{u}^{n}(K)$ and $\theta_{u, J}^{n-1}=\theta_{u, J}^{n}=\theta_{v, J}^{m-1}=-1$. So we can apply Lemma 5.7 and we get that $J \in \mathcal{U}_{u}^{n-1}(K)$. Contradiction.

\section{Proof of Lemma 5.4}

The proof is very similar to the one of Lemma 5.3, but there are some additional cases. For the reader's convenience we give all the structure of the proof. By contradiction, assume that $\theta_{v}^{m} \nsucceq \theta_{u}^{n}$. Then, there exists $J$ such that

$$
\left\{\begin{array}{l}
\theta_{v, J}^{m}<\theta_{u, J}^{n} \\
\text { or } \\
\theta_{v, J}^{m}=\theta_{u, J}^{n}=\sigma_{J} \text { and } \exists K: J \in \mathcal{U}_{v}^{m}(K) \cap \mathcal{U}_{u}^{n}(K) \text { and } \sigma_{J} u_{J \rightarrow K}^{n}<\sigma_{J} v_{J \rightarrow K}^{m}
\end{array}\right.
$$

The proof is decomposed in several cases:

Case 1: $J \in N A_{u}^{n} \backslash N A_{v}^{m}$

Sub-case 1.1: $\theta_{v, J}^{m-1}>\theta_{u, J}^{n-1}$

Then $\theta_{v, J}^{m-1}=1$ and $\theta_{u, J}^{n-1}=-1$. Since $J \in N A_{u}^{n} \backslash N A_{v}^{m}$, we deduce that $\theta_{u, J}^{n}=1=\theta_{v, J}^{m}=\sigma_{J}$. We then have

$$
v_{J \rightarrow K}^{m} \leq s_{m}=t_{n}=u_{J \rightarrow K}^{n}
$$

where we have used the fact that $J \in \mathcal{U}_{v}^{m}(K)$ and Proposition 4.1 Point 2 for the first inequality and the fact that $J \in N A_{u}^{n} \cap \mathcal{U}_{u}^{n}(K)$ joint to Proposition 4.1 Point 4 for the last equality. This contradicts (5.8). 
Sub-case 1.2: $\theta_{v, J}^{m-1}=\theta_{u, J}^{n-1}$

Sub-case 1.2.1: $\theta_{v, J}^{m-1}=\theta_{u, J}^{n-1}=1$

Since $J \in N A_{u}^{n} \backslash N A_{v}^{m}$, we deduce that $\theta_{u, J}^{n}=-1$ and $\theta_{v, J}^{m}=1$. This contradicts (5.8).

Sub-case 1.2.2: $\theta_{v, J}^{m-1}=\theta_{u, J}^{n-1}=-1$

Step 1: $\tilde{u}_{J}^{n-1} \geq \tilde{v}_{J}^{m-1}$

Since $J \in N A_{u}^{n}$ and $\theta_{u, J}^{n-1}=-1$, we deduce that $J \in N B_{u,-}^{n-1}$. Moreover $\left|t_{n-1}-s_{m-1}\right| \leq \Delta t$, so we can apply Lemma 5.6 to get

$$
J \in N B_{v,-}^{m-1} \quad \text { and } \quad \tilde{u}_{J}^{n-1} \geq \tilde{v}_{J}^{m-1}
$$

Step 2: $\tilde{v}_{J}^{m-1}>s_{m}$

Recall that $s_{m}=\max \left(s_{m-1}, \min \left(s_{m-1}+\Delta t, \tilde{s}_{m}\right)\right)$. But $s_{m}>s_{m-1}$ and so

$$
s_{m}=\min \left(s_{m-1}+\Delta t, \tilde{s}_{m}\right) .
$$

Recall that by definition of $\tilde{s}_{m}$, we have $\tilde{s}_{m} \leq \tilde{v}_{J}^{m-1}$ with $J \in N B_{v,-}^{m-1}$. We distinguish two cases:

1. $\tilde{v}_{J}^{m-1}>\tilde{s}_{m}$. Then

$$
s_{m} \leq \tilde{s}_{m}<\tilde{v}_{J}^{m-1}
$$

2. $\tilde{v}_{J}^{m-1}=\tilde{s}_{m}$. Since $J \notin N A_{v}^{m}$, we deduce that (see Step 7 and 8 of the algorithm)

$$
s_{m}=s_{m-1}+\Delta t<\tilde{s}_{m}=\tilde{v}_{J}^{m-1} .
$$

\section{Step 3: Contradiction}

By Step 1 and 2, we get

$$
\tilde{u}_{J}^{n-1} \geq \tilde{v}_{J}^{m-1}>s_{m}=t_{n}
$$

This is impossible because $J \in N A_{u}^{n}$ (see Proposition 4.1 Point 5).

Sub-case 1.3: $\theta_{v, J}^{m-1}<\theta_{u, J}^{n-1}$

This is impossible since $\theta_{v}^{m-1} \succeq \theta_{u}^{n-1}$.

Case 2: $J \in N A_{v}^{m} \backslash N A_{u}^{n}$

By symmetry (see Proposition 4.3), this case can be treated in the same way of Case 1.

Case 3: $J \in N A_{u}^{n} \cap N A_{v}^{m}$

Sub-case 3.1: $\theta_{v, J}^{m-1}>\theta_{u, J}^{n-1}$

This implies that $\theta_{v, J}^{m-1}=1$ and $\theta_{u, J}^{n-1}=-1$. Using also that $J \in N A_{u}^{n} \cap N A_{v}^{m}$, we deduce that $J \in$ $N B_{u,-}^{n-1} \cap N B_{v,+}^{m-1}$

This implies that

$$
\hat{c}_{u, J}^{n-1}>0 \text { and } \hat{c}_{v, J}^{m-1}<0 .
$$

This is absurd since $\left|s_{m-1}-t_{n-1}\right|<\Delta t$.

Sub-case 3.2: $\theta_{v, J}^{m-1}=\theta_{u, J}^{n-1}$

Since $J \in N A_{u}^{n} \cap N A_{v}^{m}$, we deduce that $\theta_{u, J}^{n}=\theta_{v, J}^{m}$. Using that $J \in \mathcal{U}_{u}^{n}(K) \cap \mathcal{U}_{v}^{m}(K)$, we have by Proposition 4.1 point 4

$$
v_{J \rightarrow K}^{m}=s_{m}=t_{n}=u_{J \rightarrow K}^{n} .
$$

This contradicts (5.8).

Sub-case 3.3: $\theta_{v, J}^{m-1}<\theta_{u, J}^{n-1}$

This is impossible since $\theta_{v}^{m-1} \succeq \theta_{u}^{n-1}$.

Case 4: $J \notin N A_{u}^{n} \cup N A_{v}^{m}$

Sub-case 4.1: $\theta_{v, J}^{m}<\theta_{u, J}^{n}$

Since $J \notin N A_{u}^{n} \cup N A_{v}^{m}$, we have $\theta_{u, J}^{n-1}=\theta_{u, J}^{n}$ and $\theta_{v, J}^{m-1}=\theta_{v, J}^{m}$. We then get $\theta_{v, J}^{m-1}<\theta_{u, J}^{n-1}$. Contradiction with $\theta_{v}^{m-1} \succeq \theta_{u}^{n-1}$. 
Sub-case 4.2: $\theta_{v, J}^{m}=\theta_{u, J}^{n}$

Since $J \notin N A_{u}^{n} \cup N A_{v}^{m}$, we have

$$
\theta_{u, J}^{n-1}=\theta_{u, J}^{n}=\theta_{v, J}^{m-1}=\theta_{v, J}^{m}=\sigma_{J}= \pm 1 .
$$

From (5.8), there exists $K$ such that $J \in \mathcal{U}_{v}^{m}(K) \cap \mathcal{U}_{u}^{n}(K)$ and

$$
\sigma_{J} u_{J \rightarrow K}^{n}<\sigma_{J} v_{J \rightarrow K}^{m}
$$

We then distinguish several sub-cases:

Sub-case 4.2.1: $J \in \mathcal{U}_{u}^{n-1}(K) \cap \mathcal{U}_{v}^{m-1}(K)$

From (5.9) and the fact that $\theta_{v}^{m-1} \succeq \theta_{u}^{n-1}$, we have

$$
\sigma_{J} u_{J \rightarrow K}^{n-1} \geq \sigma_{J} v_{J \rightarrow K}^{m-1}
$$

Now $J \in \mathcal{U}_{u}^{n-1}(K) \cap \mathcal{U}_{u}^{n}(K)$ and $J \in \mathcal{U}_{v}^{m-1}(K) \cap \mathcal{U}_{v}^{m}(K)$ imply, by Proposition 4.1 Point 6, that $u_{J \rightarrow K}^{n}=$ $u_{J \rightarrow K}^{n-1}$ and $v_{J \rightarrow K}^{m}=v_{J \rightarrow K}^{m-1}$. Therefore $\sigma_{J} u_{J \rightarrow K}^{n} \geq \sigma_{J} v_{J \rightarrow K}^{m}$, which contradicts (5.10).

Sub-case 4.2.2: $J \in \mathcal{U}_{v}^{m-1}(K) \backslash \mathcal{U}_{u}^{n-1}(K)$

Sub-case 4.2.2.1: $\sigma_{J}=+1$

Using the fact that $J \in \mathcal{U}_{u}^{n}(K) \backslash \mathcal{U}_{u}^{n-1}(K)$, joint to Proposition 4.1 Point 7, we get that $u_{J \rightarrow K}^{n}=t_{n}$. This implies that

$$
u_{J \rightarrow K}^{n}=t_{n}=s_{m} \geq v_{J \rightarrow K}^{m}
$$

where we have used the fact that $J \in \mathcal{U}_{v}^{m}(K)$ and Proposition 4.1 Point 2. This contradicts (5.10).

Sub-case 4.2.2.2: $\sigma_{J}=-1$

In this case, we have $J \in \mathcal{U}_{v}^{m-1}(K) \cap \mathcal{U}_{u}^{n}(K)$ and $\theta_{u, J}^{n-1}=\theta_{u, J}^{n}=\theta_{v, J}^{m-1}=-1$. So we can apply Lemma 5.7 and we get that $J \in \mathcal{U}_{u}^{n-1}(K)$. Contradiction.

Sub-case 4.2.3: $J \in \mathcal{U}_{u}^{n-1}(K) \backslash \mathcal{U}_{v}^{m-1}(K)$

By symmetry, this sub-case can be treated in the same way of sub-case 4.2.2.

Sub-case 4.2.4: $J \notin \mathcal{U}_{u}^{n-1}(K) \cup \mathcal{U}_{v}^{m-1}(K)$

Since $J \in \mathcal{U}_{u}^{n}(K) \backslash \mathcal{U}_{u}^{n-1}(K)$ and $J \in \mathcal{U}_{v}^{m}(K) \backslash \mathcal{U}_{v}^{v-1}(K)$, we deduce by Proposition 4.1 Point 7 that

$$
v_{J \rightarrow K}^{m}=s_{m}=t_{n}=u_{J \rightarrow K}^{n} .
$$

This contradicts (5.10).

\section{Comparison principle for the $\tilde{t}_{n}$ times: proof of Proposition 5.5}

This subsection is devoted to the proof of Proposition 5.5. We will prove a more general result (see Proposition 6.2 . We recall that we consider two sequences

$$
t_{n-p-1}<t_{n-p}=\ldots=t_{n}<t_{n+1} \quad \text { and } \quad s_{m-q-1}<s_{m-q}=\ldots=s_{m}<s_{m+1}
$$

such that $t_{n}=s_{m} \leq T$. For simplicity of notation, we redefine, only in this subsection,

$$
\tilde{t}_{n-p}=-\infty \quad \text { and } \quad \tilde{s}_{m-q}=-\infty .
$$

We recall that by definition of comparable time Definition 3.1, that $\tilde{t}_{n-k}$ and $\tilde{s}_{m-l}$ are comparable for $0 \leq k \leq p$ and $0 \leq l \leq q$ if

$$
\left(C\left(\tilde{t}_{n-k}, \tilde{s}_{m-l}\right)\right) \quad \begin{cases} & \tilde{s}_{m-l} \leq \tilde{t}_{n-k}<\tilde{s}_{m-l+1} \\
\text { or } \quad \begin{array}{l}
\tilde{t}_{n-k} \leq \tilde{s}_{m-l}<\tilde{t}_{n-k+1}
\end{array}\end{cases}
$$


Remark 6.1 Let us point out that for $0 \leq k \leq p$ (resp. $0 \leq l \leq q)$, we always have

$$
\tilde{t}_{n-k}<\tilde{t}_{n-k+1} \quad\left(\operatorname{resp} . \tilde{s}_{m-l}<\tilde{s}_{m-l+1}\right) \text {. }
$$

This comes from the fact that the velocity $c_{u}^{n-k}\left(\right.$ resp. $\left.c_{v}^{m-l}\right)$ is independent on $k=0, \ldots, p($ resp. $l=0, \ldots, q)$ and from our definition (6.1).

To prove Proposition 5.5, we will prove the following more general result:

Proposition 6.2 (Comparison principle for the $\tilde{t}_{n}$ times)

For $p, q \geq 0$, if $\theta_{v}^{m-q} \succeq \theta_{u}^{n-p}$ for $p+q \geq 1$, then $\theta_{v}^{m-l} \succeq \theta_{u}^{n-k}$ for $0 \leq k \leq p, 0 \leq l \leq q$ and for every times satisfying $C\left(\tilde{t}_{n-k}, \tilde{s}_{m-l}\right)$.

Remark 6.3 We have $\tilde{s}_{m} \leq \tilde{t}_{n} \leq t_{n}=s_{m}<s_{m+1} \leq \tilde{s}_{m+1}$ or $\tilde{t}_{n} \leq \tilde{s}_{m} \leq s_{m}=t_{n}<t_{n+1} \leq \tilde{t}_{n+1}$ (see Proposition 4.1 Point 8 and 9). This implies that $C\left(\overline{\tilde{t}}_{n}, \tilde{s}_{m}\right)$ is true and then Proposition 6.2 implies that $\theta_{v}^{m} \succeq \theta_{u}^{n}$ and so Proposition 5.5 is true.

The proof of Proposition 6.2 is similar to the one of Theorem 5.1 and we need the equivalent of Lemma 5.3 and 5.4 whose proofs are postponed:

Lemma 6.4 (Two jumps, two arrivals)

Assume that $\theta_{v}^{m-l} \succeq \theta_{u}^{n-k}$ for $0 \leq k \leq p, 0 \leq l \leq q$ and $l+k \geq 1$. If $\tilde{s}_{m-l} \leq \tilde{t}_{n-k+1}<\tilde{s}_{m-l+1}$ (resp. $\left.\tilde{t}_{n-k} \leq \tilde{s}_{m-l+1}<\tilde{t}_{n-k+1}\right)$, then $\theta_{v}^{m-l} \succeq \theta_{u}^{n-k+1}$ (resp. $\left.\theta_{v}^{m-l+1} \succeq \theta_{u}^{n-k}\right)$.

Lemma 6.5 (Two jumps, one arrival)

Assume that $\theta_{v}^{m-l} \succeq \theta_{u}^{n-k}$ for $1 \leq k \leq p, 1 \leq l \leq q$. If $\tilde{s}_{m-l+1}=\tilde{t}_{n-k+1}$, then $\theta_{v}^{m-l+1} \succeq \theta_{u}^{n-k+1}$.

\section{Proof of Proposition 6.2}

By contradiction, let us define

$$
\tilde{s}^{*}=\inf \left\{\begin{array}{l}
\min \left(\tilde{t}_{n-k}, \tilde{s}_{m-l}\right) \text { such that } \theta_{v}^{m-l} \nsucceq \theta_{u}^{n-k} \\
0 \leq k \leq p, 0 \leq l \leq q \text { and } C\left(\tilde{t}_{n-k}, \tilde{s}_{m-l}\right) \text { is true }
\end{array}\right\}
$$

Up to a symmetry (see Proposition 4.3 ), we can assume that $\tilde{s}^{*}=\tilde{s}_{m-l^{*}}$ for a certain index $l^{*}$. Then let us define

$$
k^{*}=\sup \left\{0 \leq k \leq p, ; \text { such that } \theta_{v}^{m-l^{*}} \nsucceq \theta_{u}^{n-k}, C\left(\tilde{t}_{n-k}, \tilde{s}_{m-l^{*}}\right) \text { is true }\right\}
$$

Therefore $\left(n-k^{*}, m-l^{*}\right)$ is the minimal couple such that $\theta_{v}^{m-l^{*}} \nsucceq \theta_{u}^{n-k^{*}}$ with

$$
\tilde{s}_{m-l^{*}} \leq \tilde{t}_{n-k^{*}}<\tilde{s}_{m-l^{*}+1} .
$$

Since $C\left(\tilde{t}_{n-p}, \tilde{s}_{m-q}\right)$ is true, we get that $k^{*}<p$ or $l^{*}<q$ and since $\tilde{s}_{m-l^{*}} \leq \tilde{t}_{n-k^{*}}$, we get that $k^{*}<p$.

To simplify the notation, let us denote $k^{*}$ by $k$ and $l^{*}$ by $l$. The proof is decomposed into two cases:

Case 1: $\tilde{s}_{m-l}<\tilde{t}_{n-k}$

Step $1: \theta_{v}^{m-l} \succeq \theta_{u}^{n-k-1}$

To prove that $\theta_{v}^{m-l} \succeq \theta_{u}^{n-k-1}$ is true, it suffices to show that $C\left(t_{n-k-1}, s_{m-1}\right)$ ) is satisfied (because ( $n-$ $k, m-l)$ is the minimal couple such that $\left.\theta_{v}^{m-l} \nsucceq \theta_{u}^{n-k}\right)$. We recall that

$$
\tilde{s}_{m-l}<\tilde{t}_{n-k}<\tilde{s}_{m-l+1} .
$$
$\tilde{t}_{n-k}$.

If $\tilde{s}_{m-l} \leq \tilde{t}_{n-k-1}$, then $\tilde{s}_{m-l} \leq \tilde{t}_{n-k-1}<\tilde{t}_{n-k}<\tilde{s}_{m-l+1}$, while if $\tilde{t}_{n-k-1}<\tilde{s}_{m-l}$, then $\tilde{t}_{n-k-1}<\tilde{s}_{m-l}<$

So, we always have that property $\left(C\left(\tilde{t}_{n-k-1}, \tilde{s}_{m-l}\right)\right)$ is satisfied, which implies that $\theta_{v}^{m-l} \succeq \theta_{u}^{n-k-1}$.

\section{Step 2: Contradiction.}

Using Lemma 6.4, we deduce that $\theta_{v}^{m-l} \succeq \theta_{u}^{n-k}$. Contradiction.

Case 2: $\tilde{s}_{m-l}=\tilde{t}_{n-k}$

Since $k<p$, we deduce that $\tilde{s}_{m-l}=\tilde{t}_{n-k}>-\infty$ and so $l<q$. Moreover, we have $\tilde{t}_{n-k-1} \leq \tilde{s}_{m-l-1}<$ $\tilde{s}_{m-l}=\tilde{t}_{n-k}$ or $\tilde{s}_{m-l-1} \leq \tilde{t}_{n-k-1}<\tilde{t}_{n-k}=\tilde{s}_{m-l}$ and so $C\left(\tilde{t}_{n-k-1}, \tilde{s}_{m-l-1}\right)$ is satisfied. Therefore, this implies (by definition of $(k, l))$ that $\theta_{v}^{m-l-1} \succeq \theta_{u}^{n-k-1}$. Using Lemma 6.5 , we deduce that $\theta_{v}^{m-l} \succeq \theta_{u}^{n-k}$. Contradiction. 
We now give the proofs of Lemma 6.4 and 6.5.

\section{Proof of Lemma 6.4}

We make the proof in the case $\tilde{s}_{m-l+1}>\tilde{t}_{n-k+1}$, the other case being equivalent by symmetry (see Proposition 4.3). Since $\tilde{t}_{n+1} \geq t_{n+1}>t_{n}=s_{m} \geq \tilde{s}_{m} \geq \tilde{s}_{m-l+1}$ for $1 \leq l \leq q+1$ (see Proposition 4.1 Point 8 for the first inequality and Point 9 for the last one), we deduce that $1 \leq k \leq p$ and $t_{n-k+1}=t_{n}$.

By contradiction, assume that $\theta_{v}^{m-l} \nsucceq \theta_{u}^{n-k+1}$. Then there exists $J$ such that

$$
\left\{\begin{array}{l}
\theta_{v, J}^{m-l}<\theta_{u, J}^{n-k+1} \\
\text { or } \\
\theta_{v, J}^{m-l}=\theta_{u, J}^{n-k+1}=\sigma_{J} \text { and } \exists K: J \in \mathcal{U}_{v}^{m-l}(K) \cap \mathcal{U}_{u}^{n-k+1}(K) \text { and } \sigma_{J} u_{J \rightarrow K}^{n-k+1}<\sigma_{J} v_{J \rightarrow K}^{m-l}
\end{array}\right.
$$

The proof is decomposed in several cases:

Case 1: $J \in N A_{u}^{n-k+1}$

Sub-case 1.1: $\theta_{v, J}^{m-l}>\theta_{u, J}^{n-k}$

Then $\theta_{v, J}^{m-l}=1$ and $\theta_{u, J}^{n-k}=-1$. Since $J \in N A_{u}^{n-k+1}$, we deduce that $\theta_{u, J}^{n-k+1}=1=\theta_{v, J}^{m-l}=\sigma_{J}$. We then have

$$
v_{J \rightarrow K}^{m-l} \leq s_{m-l}=t_{n-k+1}=u_{J \rightarrow K}^{n-k+1}
$$

where we have used the fact that $J \in \mathcal{U}_{v}^{m-l}(K)$ and Proposition 4.1 Point 2 for the first inequality and the fact that $J \in N A_{u}^{n-k+1} \cap \mathcal{U}_{u}^{n-k+1}(K)$ joint to Proposition 4.1 Point 4 for the last equality. This contradicts (6.2).

Sub-case 1.2: $\theta_{v, J}^{m-l}=\theta_{u, J}^{n-k}$

Sub-case 1.2.1: $\theta_{v, J}^{m-l}=\theta_{u, J}^{n-k}=1$

Since $J \in N A_{u}^{n-k+1}$, we deduce that $\theta_{u, J}^{n-k+1}=-1$. This contradicts (6.2).

Sub-case 1.2.2: $\theta_{v, J}^{m-l}=\theta_{u, J}^{n-k}=-1$

Step 1: $\tilde{u}_{J}^{n-k} \geq \tilde{v}_{J}^{m-l}$

Since $t_{n-k}=s_{m-l}$, we can apply Lemma 5.6 (recall that $J \in N A_{u}^{n-k+1}$ with $\theta_{u, J}^{n-k}=-1$ and so $J \in N B_{u,-}^{n-k}$ and by assumption in Lemma 6.4, we have $\theta_{v}^{m-l} \succeq \theta_{u}^{n-k}$ ) to get

$$
\tilde{u}_{J}^{n-k} \geq \tilde{v}_{J}^{m-l}
$$

\section{Step 2: Contradiction}

Since $J \in N A_{u}^{n-k+1}$, we know that $\tilde{t}_{n-k+1}=\tilde{u}_{J}^{n-k}$. By Step 1, we then get

$$
\tilde{t}_{n-k+1}=\tilde{u}_{J}^{n-k} \geq \tilde{v}_{J}^{m-l} \geq \tilde{s}_{m-l+1}
$$

where we have used the definition of $\tilde{s}_{m-l+1}$ (see point 5 of the algorithm) for the last inequality. This contradicts the fact that $\tilde{s}_{m-l+1}>\tilde{t}_{n-k+1}$

Sub-case 1.3: $\theta_{v, J}^{m-l}<\theta_{u, J}^{n-k}$

This is impossible since $\theta_{v}^{m-l} \succeq \theta_{u}^{n-k}$.

Case 2: $J \notin N A_{u}^{n-k+1}$

Sub-case 2.1: $\theta_{v, J}^{m-l}<\theta_{u, J}^{n-k+1}$

Since $J \notin N A_{u}^{n-k+1}$, we have $\theta_{u, J}^{n-k+1}=\theta_{u, J}^{n-k}$ and so $\theta_{v, J}^{m-l}<\theta_{u, J}^{n-k}$. Contradiction with $\theta_{v}^{m-l} \succeq \theta_{u}^{n-k}$.

Sub-case 2.2: $\theta_{v, J}^{m-l}=\theta_{u, J}^{n-k+1}$

Since $J \notin N A_{u}^{n-k+1}$, we have

$$
\theta_{u, J}^{n-k}=\theta_{u, J}^{n-k+1}=\theta_{v, J}^{m-l}=\sigma_{J}= \pm 1 .
$$

From (6.2), there exists $K$ such that $J \in \mathcal{U}_{v}^{m-l}(K) \cap \mathcal{U}_{u}^{n-k+1}(K)$ and

$$
\sigma_{J} u_{J \rightarrow K}^{n-k+1}<\sigma_{J} v_{J \rightarrow K}^{m-l} .
$$


We then distinguish several sub-cases:

Sub-case 2.2.1: $J \in \mathcal{U}_{u}^{n-k}(K)$

From (6.3) and the fact that $\theta_{v}^{m-l} \succeq \theta_{u}^{n-k}$, we have

$$
\sigma_{J} u_{J \rightarrow K}^{n-k} \geq \sigma_{J} v_{J \rightarrow K}^{m-l}
$$

Now $J \in \mathcal{U}_{u}^{n-k}(K) \cap \mathcal{U}_{u}^{n-k+1}(K)$ imply, by Proposition 4.1 Point 6 , that $u_{J \rightarrow K}^{n-k+1}=u_{J \rightarrow K}^{n-k}$. Therefore $\sigma_{J} u_{J \rightarrow K}^{n-k+1} \geq \sigma_{J} v_{J \rightarrow K}^{m-l}$, which contradicts (6.4).

Sub-case 2.2.2: $J \notin \mathcal{U}_{u}^{n-k}(K)$ and $\sigma_{J}=+1$

Using the fact that $J \in \mathcal{U}_{u}^{n-k+1}(K) \backslash \mathcal{U}_{u}^{n-k}(K)$, joint to Proposition 4.1 Point 7, we get that $u_{J \rightarrow K}^{n-k+1}=t_{n-k+1}$. This implies that

$$
u_{J \rightarrow K}^{n-k+1}=t_{n-k+1}=s_{m-l} \geq v_{J \rightarrow K}^{m-l}
$$

where we have used the fact that $J \in \mathcal{U}_{v}^{m-l}(K)$ joint to Proposition 4.1 Point 2 for the last inequality. Contradiction with (6.4).

Sub-case 2.2.3: $J \notin \mathcal{U}_{u}^{n-k}(K)$ and $\sigma_{J}=-1$

In this case, we have $J \in \mathcal{U}_{v}^{m-l}(K) \cap \mathcal{U}_{u}^{n-k+1}(K)$ and $\theta_{u, J}^{n-k}=\theta_{u, J}^{n-k+1}=\theta_{v, J}^{m-l}=-1$. So we can apply Lemma 5.7 and we get that $J \in \mathcal{U}_{u}^{n-k}(K)$. Contradiction.

\section{Proof of Lemma 6.5}

By contradiction, assume that $\theta_{v}^{m-l+1} \nsucceq \theta_{u}^{n-k+1}$. Then, there exists $J$ such that

$$
\left\{\begin{array}{l}
\theta_{v, J}^{m-l+1}<\theta_{u, J}^{n-k+1} \\
\text { or } \\
\theta_{v, J}^{m-l+1}=\theta_{u, J}^{n-k+1}=\sigma_{J} \text { and } \exists K \backslash J \in \mathcal{U}_{v}^{m-l+1}(K) \cap \mathcal{U}_{u}^{n-k+1}(K) \text { and } \sigma_{J} u_{J \rightarrow K}^{n-k+1}<\sigma_{J} v_{J \rightarrow K}^{m-l+1}
\end{array}\right.
$$

The proof is decomposed in several cases:

Case 1: $J \in N A_{u}^{n-k+1} \backslash N A_{v}^{m-l+1}$

Sub-case 1.1: $\theta_{v, J}^{m-l}>\theta_{u, J}^{n-k}$

Then $\theta_{v, J}^{m-l}=1$ and $\theta_{u, J}^{n-k}=-1$. Since $J \in N A_{u}^{n-k+1} \backslash N A_{v}^{m-l+1}$, we deduce that $\theta_{u, J}^{n-k+1}=1=\theta_{v, J}^{m-l+1}=\sigma_{J}$. We then have

$$
v_{J \rightarrow K}^{m-l+1} \leq s_{m-l+1}=t_{n-k+1}=u_{J \rightarrow K}^{n-k+1}
$$

where we have used the fact that $J \in \mathcal{U}_{v}^{m-l+1}(K)$ and Proposition 4.1 Point 2 for the first inequality and the fact that $J \in N A_{u}^{n-k+1} \cap \mathcal{U}_{u}^{n-k+1}(K)$ joint to Proposition 4.1 Point 4 for the last equality. This contradicts (6.5).

Sub-case 1.2: $\theta_{v, J}^{m-l}=\theta_{u, J}^{n-k}$

Sub-case 1.2.1: $\theta_{v, J}^{m-l}=\theta_{u, J}^{n-k}=1$

Since $J \in N A_{u}^{n-k+1} \backslash N A_{v}^{m-l+1}$, we deduce that $\theta_{u, J}^{n-k+1}=-1$ and $\theta_{v, J}^{m-l+1}=1$. This contradicts (6.5).

Sub-case 1.2.2: $\theta_{v, J}^{m-l}=\theta_{u, J}^{n-k}=-1$

Step 1: $\tilde{u}_{J}^{n-k} \geq \tilde{v}_{J}^{m-l}$

Since $J \in N A_{u}^{n-k+1}$ with $\theta_{u, J}^{n-k}=-1$, we deduce that $J \in N B_{u,-}^{n-k}$. Moreover $t_{n-k}=s_{m-l}$ joint to the fact that by assumption in Lemma 6.5 , we have $\theta_{v}^{m-l} \succeq \theta_{u}^{n-k}$, imply that we can apply Lemma 5.6 to get

$$
\tilde{u}_{J}^{n-k} \geq \tilde{v}_{J}^{m-l}
$$

\section{Step 2: Contradiction}

Because $s_{m-l+1}=s_{m-l}$ and $J \notin N A_{v}^{m}$, we deduce (see Point 8 of the algorithm) that $\tilde{s}_{m-l+1}<\tilde{v}_{J}^{m-l}$. By Step 1, we then get

$$
\tilde{u}_{J}^{n-k} \geq \tilde{v}_{J}^{m-l}>\tilde{s}_{m-l+1}=\tilde{t}_{n-k+1} .
$$

This is impossible because $J \in N A_{u}^{n-k+1}$ (see Point 8 of the algorithm).

Sub-case 1.3: $\theta_{v, J}^{m-l}<\theta_{u, J}^{n-k}$

This is impossible since $\theta_{v}^{m-l} \succeq \theta_{u}^{n-k}$. 
Case 2: $J \in N A_{v}^{m} \backslash N A_{u}^{n}$

By symmetry (see Proposition 4.3), this case can be treated in the same way of case 1 .

Case 3: $J \in N A_{u}^{n-k+1} \cap N A_{v}^{m-l+1}$

Sub-case 3.1: $\theta_{v, J}^{m-l}>\theta_{u, J}^{n-k}$

This implies that $\theta_{v, J}^{m-l}=1$ and $\theta_{u, J}^{n-k}=-1$. Using also that $J \in N A_{u}^{n-k+1} \cap N A_{v}^{m-l+1}$, we deduce that $J \in N B_{u,-}^{n-k} \cap N B_{v,+}^{m-l}$

This implies that

$$
\hat{c}_{u, J}^{n-k}>0 \text { and } \quad \hat{c}_{v, J}^{m-l}<0 .
$$

This is absurd since $s_{m-l}=t_{n-k}$.

Sub-case 3.2: $\theta_{v, J}^{m-l}=\theta_{u, J}^{n-k}$

Since $J \in N A_{u}^{n-k+1} \cap N A_{v}^{m-l+1}$, we deduce that $\theta_{u, J}^{n-k+1}=\theta_{v, J}^{m-l+1}$. Using that $J \in \mathcal{U}_{u}^{n-k+1}(K) \cap \mathcal{U}_{v}^{m-l+1}(K)$, we have by Proposition 4.1 point 4

$$
v_{J \rightarrow K}^{m-l+1}=s_{m-l+1}=t_{n-k+1}=u_{J \rightarrow K}^{n-k+1} .
$$

This contradicts (6.5).

Sub-case 3.3: $\theta_{v, J}^{m-l}<\theta_{u, J}^{n-k}$

This is impossible since $\theta_{v}^{m-l} \succeq \theta_{u}^{n-k}$.

Case 4: $J \notin N A_{u}^{n-k+1} \cup N A_{v}^{m-l+1}$

Sub-case 4.1: $\theta_{v, J}^{m-l+1}<\theta_{u, J}^{n-k+1}$

Since $J \notin N A_{u}^{n-k+1} \cup N A_{v}^{m-l+1}$, we have $\theta_{u, J}^{n-k}=\theta_{u, J}^{n-k+1}$ and $\theta_{v, J}^{m-l}=\theta_{v, J}^{m-l+1}$. We then get $\theta_{v, J}^{m-l}<\theta_{u, J}^{n-k}$. Contradiction with $\theta_{v}^{m-l} \succeq \theta_{u}^{n-k}$.

Sub-case 4.2: $\theta_{v, J}^{m-l+1}=\theta_{u, J}^{n-k+1}$

Since $J \notin N A_{u}^{n-k+1} \cup N A_{v}^{m-l+1}$, we have

$$
\theta_{u, J}^{n-k}=\theta_{u, J}^{n-k+1}=\theta_{v, J}^{m-l}=\theta_{v, J}^{m-l+1}=\sigma_{J}= \pm 1 .
$$

From (6.5), there exists $K$ such that $J \in \mathcal{U}_{v}^{m-l+1}(K) \cap \mathcal{U}_{u}^{n-k+1}(K)$ and

$$
\sigma_{J} u_{J \rightarrow K}^{n-k+1}<\sigma_{J} v_{J \rightarrow K}^{m-l+1}
$$

We then distinguish several sub-cases:

Sub-case 4.2.1: $J \in \mathcal{U}_{u}^{n-k}(K) \cap \mathcal{U}_{v}^{m-l}(K)$

From (6.6) and the fact that $\theta_{v}^{m-l} \succeq \theta_{u}^{n-k}$, we have

$$
\sigma_{J} u_{J \rightarrow K}^{n-k} \geq \sigma_{J} v_{J \rightarrow K}^{m-l}
$$

Now $J \in \mathcal{U}_{u}^{n-k}(K) \cap \mathcal{U}_{u}^{n-k+1}(K)$ and $J \in \mathcal{U}_{v}^{m-l}(K) \cap \mathcal{U}_{v}^{m-l+1}(K)$ imply, by Proposition 4.1 Point 6 , that $u_{J \rightarrow K}^{n-k+1}=u_{J \rightarrow K}^{n-k}$ and $v_{J \rightarrow K}^{m-l+1}=v_{J \rightarrow K}^{m-l}$. Therefore $\sigma_{J} u_{J \rightarrow K}^{n-k+1} \geq \sigma_{J} v_{J \rightarrow K}^{m-l+1}$, which contradicts (6.7).

Sub-case 4.2.2: $J \in \mathcal{U}_{v}^{m-l}(K) \backslash \mathcal{U}_{u}^{n-k}(K)$

Sub-case 4.2.2.1: $\sigma_{J}=+1$

Using the fact that $J \in \mathcal{U}_{u}^{n-k+1}(K) \backslash \mathcal{U}_{u}^{n-k}(K)$, joint to Proposition 4.1 Point 7, we get that $u_{J \rightarrow K}^{n-k+1}=t_{n-k+1}$. This implies that

$$
u_{J \rightarrow K}^{n-k+1}=t_{n-k+1}=s_{m-l+1} \geq v_{J \rightarrow K}^{m-l+1}
$$

where we have used the fact that $J \in \mathcal{U}_{v}^{m-l+1}(K)$ and Proposition 4.1 Point 2 for the last inequality. This contradicts (6.7).

Sub-case 4.2.2.2: $\sigma_{J}=-1$

In this case, we have $J \in \mathcal{U}_{v}^{m-l}(K) \cap \mathcal{U}_{u}^{n-k+1}(K)$ and $\theta_{u, J}^{n-k}=\theta_{u, J}^{n-k+1}=\theta_{v, J}^{m-l}=-1$. So we can apply Lemma 5.7 and we get that $J \in \mathcal{U}_{u}^{n-k}(K)$. Contradiction.

Sub-case 4.2.3: $J \in \mathcal{U}_{u}^{n-k}(K) \backslash \mathcal{U}_{v}^{m-l}(K)$

By symmetry, this sub-case can be treated in the same way of sub-case 4.2.2. 
Sub-case 4.2.4: $J \notin \mathcal{U}_{u}^{n-k}(K) \cup \mathcal{U}_{v}^{m-l}(K)$

Since $J \in \mathcal{U}_{u}^{n-k+1}(K) \backslash \mathcal{U}_{u}^{n-k}(K)$ and $J \in \mathcal{U}_{v}^{m-l+1}(K) \backslash \mathcal{U}_{v}^{m-l}(K)$, we deduce by Proposition 4.1 Point 7 that $v_{J \rightarrow K}^{m-l+1}=s_{m-l+1}=t_{n-k+1}=u_{J \rightarrow K}^{n-k+1}$.

This contradicts (6.7).

\section{Convergence result}

\subsection{Proof of convergence of the modified GFMM}

This subsection is devoted to the proof of Theorem 3.6.

\section{Proof of Theorem 3.6}

The proof is very similar to the one of [4, Theorem 2.5] and just needs some adaptations essentially due to the change in the point 10 of the algorithm. The only point to check is the sub-solution property of the limit [4, Proposition 6.1]. By contradiction, we assume that there are $\left(x_{0}, t_{0}\right)$ and $\varphi \in C^{2}$ such that $\bar{\theta}^{0}-\varphi$ reaches a strict maximum at $\left(x_{0}, t_{0}\right)$ with $\bar{\theta}^{0}\left(x_{0}, t_{0}\right)=\varphi\left(x_{0}, t_{0}\right)$ and

$$
\varphi_{t}\left(x_{0}, t_{0}\right)=\alpha+c\left(x_{0}, t_{0}\right)\left|D \varphi\left(x_{0}, t_{0}\right)\right|
$$

with $\alpha>0$.

Now, the proof depends on the sign of the velocity. In the case $c\left(x_{0}, t_{0}\right)>0$ or $c\left(x_{0}, t_{0}\right)<0$, it suffices to replace [4, Lemma 5.1] by the following lemma (which proof is equivalent) and the proof is exactly the same.

\section{Lemma 7.1 (Time character of the $u$ )}

Assume there exists $\delta>0$ and $(I, n) \in \mathbb{Z}^{N} \times \mathbb{N}$ such that $c\left(x_{I}, t_{n}\right) \geq \delta>0, \theta_{I}^{n-1}=-1$ and $\theta_{I}^{n}=1$ (resp. $c\left(x_{I}, t_{n}\right) \leq-\delta<0, \theta_{I}^{n-1}=1$ and $\left.\theta_{I}^{n}=-1\right)$, then for any $J \in \mathcal{U}^{n-1}(I)$, we have for $\Delta x \leq \frac{\delta^{2}}{16 L}$

$$
u_{J \rightarrow I}^{n-1}=\sup \left\{t_{m} \leq t_{n-1}, \theta_{J}^{m-1}=-1, \theta_{J}^{p}=1, \quad \text { for } \quad m \leq p \leq n-1\right\} \quad>\quad t_{n}-\frac{4 \Delta x}{\delta}
$$

with the convention that $u_{J \rightarrow I}^{n-1}=t_{0}$ if $\theta_{J}^{p}=1$ for $0 \leq p \leq n-1$

$$
\text { (resp. } u_{J \rightarrow I}^{n-1}=\sup \left\{t_{m} \leq t_{n-1}, \theta_{J}^{m-1}=1, \theta_{J}^{p}=-1, \quad \text { for } \quad m \leq p \leq n-1\right\} \quad>\quad t_{n}-\frac{4 \Delta x}{\delta} .
$$

with the convention that $u_{J \rightarrow I}^{n-1}=t_{0}$ if $\theta_{J}^{p}=-1$ for $\left.0 \leq p \leq n-1\right)$.

Intuitively, this result comes from the fact that if the front reaches the point $I$ at step $n$ then (in the case where the velocity is positive) it has reached all the points $J \in V(I)$ such that $\theta_{J}^{n-1}=1$ at a time closed to $t_{n}$. Moreover, the point $J$ is useful for $I$ as soon as $J$ has been accepted.

Remark 7.2 This lemma implies in particular that if the velocity is positive (or negative) then the algorithm presented in Subsection 2.2 is equivalent to the one of [4].

We now treat the case $c\left(x_{0}, t_{0}\right)=0$. In this case, we have

$$
\varphi_{t}=\alpha>0 .
$$

Since the maximum of $\bar{\theta}^{0}-\varphi$ is strict, there exists $\left(x_{\varepsilon}, t_{\varepsilon}\right) \rightarrow\left(x_{0}, t_{0}\right)$ as $\varepsilon \rightarrow 0$ such that

$$
\max \left(\left(\theta^{\varepsilon}\right)^{*}-\varphi\right)=\left(\left(\theta^{\varepsilon}\right)^{*}-\varphi\right)\left(x_{\varepsilon}, t_{\varepsilon}\right) .
$$

In particular, we have $\left(\theta^{\varepsilon}\right)^{*}\left(x_{\varepsilon}, t_{\varepsilon}\right)=1$ for $\Delta x, \Delta t$ small enough. Indeed, by contradiction, suppose that $\left(\theta^{\varepsilon}\right)^{*}\left(x_{\varepsilon}, t_{\varepsilon}\right)=-1$. Using the fact that $\left(\theta^{\varepsilon}\right)^{*}$ is upper semi-continuous, we obtain $\left(\theta^{\varepsilon}\right)^{*}=-1$ a neighbourhood of $\left(x_{\varepsilon}, t_{\varepsilon}\right)$. We then deduce that $\varphi_{t}\left(x_{\varepsilon}, t_{\varepsilon}\right)=\left|D \varphi\left(x_{\varepsilon}, t_{\varepsilon}\right)\right|=0$ and so

$$
0=\varphi_{t}\left(x_{\varepsilon}, t_{\varepsilon}\right)-c\left(x_{\varepsilon}, t_{\varepsilon}\right)\left|D \varphi\left(x_{\varepsilon}, t_{\varepsilon}\right)\right| \rightarrow \varphi_{t}\left(x_{0}, t_{0}\right)-c\left(x_{0}, t_{0}\right)\left|D \varphi\left(x_{0}, t_{0}\right)\right|=\alpha
$$

This is absurd.

We now recall the following Lemma which can be found in [4, Lemma 5.3]: 


\section{Lemma 7.3 (Separation of the phases of $\theta^{\varepsilon}$ by the level set of a test function)}

Let $\varphi \in C^{2}$ in a neighbourhood $V$ of $\left(x_{0}, t_{0}\right)$ such that $\varphi_{t}\left(x_{0}, t_{0}\right)>0$. There exist $\delta_{0}>0, r>0, \tau>0$ such that if $\max _{\bar{V}}\left(\left(\theta^{\varepsilon}\right)^{*}-\varphi\right)$ is reached at $\left(x_{\varepsilon}, t_{\varepsilon}\right) \in B_{\delta_{0}}\left(x_{0}, t_{0}\right) \subset V$ with $\left(\theta^{\varepsilon}\right)^{*}\left(x_{\varepsilon}, t_{\varepsilon}\right)=1$, then there exists $\Psi_{\varepsilon} \in C^{2}\left(B_{r}\left(x_{0}\right),\left(t_{0}-\tau, t_{0}+\tau\right)\right)$ such that

(i) For all $\left(x_{J}, t_{m}\right) \in Q_{r, \tau}\left(x_{0}, t_{0}\right)=B_{r}\left(x_{0}\right) \times\left(t_{0}-\tau, t_{0}+\tau\right)$

$$
\theta^{\varepsilon}\left(x_{J}, t_{m}\right)=1 \quad \Longrightarrow \quad t_{m} \geq \Psi_{\varepsilon}\left(x_{J}\right) .
$$

(ii) There exists $\left(I, n_{k}\right) \in \mathbb{Z}^{N} \times \mathbb{N}$ such that

$$
\left(x_{\varepsilon}, t_{\varepsilon}\right) \in \bar{S}_{I}^{k}=\left[x_{I}, x_{I}+\Delta x\right] \times\left[t_{n_{k}}, t_{n_{k+1}}\right], \quad\left(\theta^{\varepsilon}\right)^{*}\left(x_{I}, t_{n_{k}}\right)=1, \quad t_{n_{k}}=\Psi_{\varepsilon}\left(x_{I}\right)
$$

and

$$
\theta_{I}^{\bar{n}}=1, \quad \theta_{I}^{m}=-1 \quad m_{0} \leq m \leq \bar{n}-1
$$

where

$$
\bar{n}=\inf \left\{n, \quad n_{k} \leq n \leq n_{k+1}-1, \quad \theta_{I}^{n}=1\right\} \quad \text { and } \quad m_{0}=\inf \left\{m, t_{m} \geq t_{0}-\tau\right\} .
$$

(iii) The following Taylor expansion holds

$$
\Psi_{\varepsilon}\left(x_{J}\right)=\Psi_{\varepsilon}\left(x_{I}\right)-\frac{D \varphi\left(x_{0}, t_{0}\right)}{\varphi_{t}\left(x_{0}, t_{0}\right)}\left(x_{J}-x_{I}\right)+(\Delta x) O\left(\Delta x+\left|x_{I}-x_{0}\right|+\left|t_{n_{k}}-t_{0}\right|\right) .
$$

We now come back to the proof of Theorem 3.6.

For all $J \in \mathcal{U}^{\bar{n}-1}(I)$ (with $\bar{n}$ defined in Lemma 7.3), we define

$$
m_{J}=\sup \left\{k \leq \bar{n}, J \notin \mathcal{U}^{k-1}(I)\right\}
$$

We distinguish two cases:

\section{There exists $J \in \mathcal{U}^{\bar{n}-1}(I)$ such that $m_{J}<m_{0}$.}

In particular, we have that $J \in \mathcal{U}^{m}(I)$ for $m_{0} \leq m \leq \bar{n}-1$, so by Proposition 4.1 Point 6 and Point 2, we have that $u_{J \rightarrow I}^{\bar{n}-1}=u_{J \rightarrow I}^{m_{0}} \leq t_{m_{0}}$. Moreover, since $J \in \mathcal{U}^{m}(I)$ and $\theta_{I}^{m}=-1$ for $m_{0} \leq m \leq \bar{n}-1$, we deduce that

$$
\theta^{\varepsilon}\left(x_{J}, t_{m_{0}}\right)=1
$$

By (7.2), we then have $t_{m_{0}} \geq \Psi_{\varepsilon}\left(x_{J}\right)$.

We now assume that $|D \varphi| \neq 0$ (the case $|D \varphi|=0$ can be treated in a similar way). Using Lemma 7.3 iii), we deduce that

$$
t_{m_{0}} \geq \Psi_{\varepsilon}\left(x_{J}\right)=t_{n_{k}}-\frac{1}{\bar{c}} \vec{n}_{0} \cdot\left(x_{J}-x_{I}\right)+(\Delta x) O\left(\Delta x+\left|x_{I}-x_{0}\right|+\left|t_{n_{k}}-t_{0}\right|\right),
$$

where $\bar{c}=\frac{\alpha}{\left|D \varphi\left(x_{0}, t_{0}\right)\right|}>0$ and $\vec{n}_{0}=\frac{D \varphi\left(x_{0}, t_{0}\right)}{\left|D \varphi\left(x_{0}, t_{0}\right)\right|}$. We then get

$$
\begin{aligned}
t_{n_{k}}-t_{m_{0}} & \leq \frac{1}{\bar{c}} \vec{n}_{0} \cdot\left(x_{J}-x_{I}\right)+(\Delta x) O\left(\Delta x+\left|x_{I}-x_{0}\right|+\left|t_{n_{k}}-t_{0}\right|\right) \\
& \leq \frac{\Delta x}{\bar{c}}+(\Delta x) O\left(\Delta x+\left|x_{I}-x_{0}\right|+\left|t_{n_{k}}-t_{0}\right|\right)
\end{aligned}
$$

Sending $\Delta x, \Delta t$ to 0 , we remark that $t_{n_{k}} \rightarrow t_{0}$ (since $t_{\varepsilon} \rightarrow t_{0}, t_{\varepsilon} \in\left[t_{n_{k}}, t_{n_{k+1}}\left[\right.\right.$ and $t_{n_{k+1}}-t_{n_{k}} \leq \Delta t$ ) and $t_{m_{0}} \rightarrow t_{0}-\tau$ (by definition of $m_{0}$ ). This implies that

$$
t_{0}-\left(t_{0}-\tau\right)=\tau \leq 0
$$

This is absurd. 
2. For all $J \in \mathcal{U}^{\bar{n}-1}(I), m_{J} \geq m_{0}$.

Using the fact that $J \in\left(\mathcal{U}^{m_{J}}(I) \cap \ldots \cap \mathcal{U}^{\bar{n}-1}(I)\right) \backslash \mathcal{U}^{m_{J}-1}(I)$ joint to Proposition 4.1 Point 6 and 7 , we get that $u_{J \rightarrow I}^{\bar{n}-1}=u_{J \rightarrow I}^{m_{J}}=t_{m_{J}}$. Moreover (since $J \in \mathcal{U}^{m}(I)$ and $\theta_{I}^{m}=-1$ for $m_{J} \leq m \leq \bar{n}-1$ ), we have that $\theta^{\varepsilon}\left(x_{J}, t_{m_{J}}\right)=1$ and so by $(7.2)$ we have $u_{J \rightarrow I}^{\bar{n}-1}=t_{m_{J}} \geq \Psi\left(x_{J}\right)$.

We now assume that $|D \varphi| \neq 0$ (the case $|D \varphi|=0$ can be treated in a similar way). Using Lemma 7.3 iii), we deduce that

$$
u_{J \rightarrow I}^{\bar{n}-1} \geq \Psi\left(x_{J}\right)=t_{n_{k}}-\frac{1}{\bar{c}} \vec{n}_{0} \cdot\left(x_{J}-x_{I}\right)+(\Delta x) O\left(\Delta x+\left|x_{I}-x_{0}\right|+\left|t_{n_{k}}-t_{0}\right|\right),
$$

where $\bar{c}=\frac{\alpha}{\left|D \varphi\left(x_{0}, t_{0}\right)\right|}>0$ and $\vec{n}_{0}=\frac{D \varphi\left(x_{0}, t_{0}\right)}{\left|D \varphi\left(x_{0}, t_{0}\right)\right|}$. Since $I \in N A^{\bar{n}}$, we know by Proposition 4.1 Point 5 that $\tilde{u}_{I}^{\bar{n}-1} \leq t_{\bar{n}}=t_{n_{k}}$. This implies that

$$
\tilde{u}_{I}^{\bar{n}-1}-u_{J \rightarrow I}^{\bar{n}-1} \leq t_{n_{k}}-u_{J \rightarrow I}^{\bar{n}-1} \leq \frac{1}{\bar{c}} \vec{n}_{0} \cdot\left(x_{J}-x_{I}\right)+(\Delta x) O\left(\Delta x+\left|x_{I}-x_{0}\right|+\left|t_{n_{k}}-t_{0}\right|\right)
$$

We first define $u_{I^{i} \rightarrow I}^{\bar{n}-1}$ for all directions $i \in \mathcal{C} \subset\{1, . ., N\}$ such that

$$
\tilde{u}_{I}^{\bar{n}-1}-u_{I^{i} \rightarrow I}^{\bar{n}-1}:=\max \left(\tilde{u}_{I}^{\bar{n}-1}-u_{I^{i,+} \rightarrow I}^{\bar{n}-1}, \tilde{u}_{I}^{\bar{n}-1}-u_{I^{i,-} \rightarrow I}^{\bar{n}-1}\right) \geq 0 .
$$

We deduce that

$$
\begin{aligned}
\left(\frac{\Delta x}{\hat{c}_{I}^{\bar{n}-1}}\right)^{2} & =\sum_{i \in \mathcal{C}}\left(\tilde{u}_{I}^{n}-u_{I^{i} \rightarrow I}^{n-1}\right)^{2} \\
& \leq\left(\frac{\Delta x}{\bar{c}}\right)^{2}+(\Delta x)^{2} O\left(\Delta x+\left|x_{I}-x_{0}\right|+\left|t_{n_{k}}-t_{0}\right|\right)
\end{aligned}
$$

i.e.

$$
\left.\frac{1}{\mid \widehat{c}_{I}^{\bar{n}}-1}\right|^{2} \leq \frac{1}{\bar{c}^{2}}+O\left(\Delta x+\left|x_{I}-x_{0}\right|+\left|t_{n_{k}}-t_{0}\right|\right)
$$

Sending $\Delta x, \Delta t$ to 0 , yields a contradiction since $\bar{c}>c\left(x_{0}, t_{0}\right)=0$.

This ends the proof of the theorem.

\section{Appendix: heuristic error estimate for monotone evolution}

We propose an error estimate in the case of the dynamics with signed speed. This is an heuristic computation, since we assume that the solution of the PDE (1.1) is a characteristic function of a smooth hyper-surface to allow us to do all the computations. This explain why we obtain an error estimate of order 1, while for this type of scheme the expected order of convergence is $1 / 2$, see [6].

In the proof we make use of a recent result by Vladimirsky, see [11], where the propagation of a front evolving with signed speed depending on time is interpreted as a static PDE for a time-dependent control problem. We consider here the case of an isotropic dynamics, but in the cited paper the general anisotropic case is analysed.

Let $\Omega_{0}$ be a bounded domain of $\mathbb{R}^{N}$ and let $u(x)$ be the minimum time to reach a point $x \in \Omega_{0}$ starting from any point of $\partial \Omega_{0}$ with the velocity $c(x, t): \bar{\Omega}_{0} \times[0, \infty) \rightarrow(-\infty, 0)$. If $c$ is Lipschitz continuous and bounded, then the minimum time can be obtained solving the following stationary PDE:

$$
\begin{cases}-c(x, u(x))|D u(x)|=1 & \text { in } \Omega_{0} \\ u(x)=0 & \text { on } \partial \Omega_{0} .\end{cases}
$$

It is known, see for instance [7], that $c(x, t)$ can be interpreted as a normal speed of a contracting front, whose position at time $t$ is the level set $u(x)=t$. Then $\theta(x, t)=1_{\{u(x)>t\}}-1_{\{u(x) \leq t\}}$ solves the eikonal equation (1.1) with speed $c(x, t)$. 
Theorem 8.1 (Heuristic error estimate for local monotone dynamics )

Let $\Omega_{0} \subset \mathbb{R}^{N}$ be a bounded open set of $\mathbb{R}^{N}$ and consider a speed $c \in W^{1, \infty}\left(\mathbb{R}^{N} \times(0, \infty)\right)$ such that $c \leq \delta<0$. We assume that $u \in C^{2}\left(\Omega_{0}\right)$ is a solution of the stationary equation (8.1) such that $\theta(x, t)=1_{\{u(x)>t\}}$ $1_{\{u(x) \leq t\}}$ is a solution of (1.1). Let us denote by $\theta^{\varepsilon}(x, t)$, for $(x, t) \in \mathbb{R}^{N} \times(0, T]$, the numerical solution of (1.1) obtained by the GFMM algorithm with $\varepsilon=(\Delta x, \Delta t)$.

Then, for $T>0$, there exists a positive constant $K_{T}$ such that

$$
\left|\theta^{\varepsilon}-\theta\right|_{L^{\infty}\left((0, T) ; L^{1}\left(\mathbb{R}^{N}\right)\right)} \leq K_{T}(\Delta x+\Delta t) .
$$

Remark 8.2 In Theorem 8.1, it is possible to assume that $u \in C^{2}$ only on a neighbourhood of $\partial \Omega_{0}$, but for simplicity of presentation and since the proof is formal, we prefer to assume that $u \in C^{2}$ in all $\Omega_{0}$.

Before giving the proof, we need the following lemma whose proof is postponed:

Lemma 8.3 (Construction of a velocity from a $\theta$ )

Let $\Omega_{0}$ be a bounded open set, $e \in \mathbb{R}, c \in W^{1, \infty}\left(\mathbb{R}^{N} \times(0, \infty)\right)$ with $c \leq \delta<0$. We denote by $\theta^{e}$ the solution of

$$
\left\{\begin{array}{lll}
\theta_{t}^{e}=(c(x, t)+e)\left|D \theta^{e}\right| & \text { in } & \mathbb{R}^{N} \times(0, \infty) \\
\theta^{e}(\cdot, 0)=1_{\Omega_{0}}-1_{\Omega_{0}^{c}} & \text { on } & \mathbb{R}^{N}
\end{array}\right.
$$

We denote by $u^{e}$ the solution of

$$
\left\{\begin{array}{lll}
-\left(c\left(x, u^{e}(x)\right)+e\right)\left|D u^{e}(x)\right|=1 & \text { in } \quad \Omega_{0} \\
u^{e}(x)=0 & \text { on } \partial \Omega_{0} .
\end{array}\right.
$$

such that $\theta^{e}(x, t)=1_{\left\{u^{e}(x)>t\right\}}-1_{\left\{u^{e}(x) \leq t\right\}}$.

Given a mesh $\left\{x_{I}=I \Delta x, I \in \mathbb{Z}^{n}\right\}$, we define a sequence of discrete increasing time $t_{n}^{e}$ by

$$
\left\{\begin{array}{l}
t_{1}^{e}=\min \left\{u^{e}\left(x_{I}\right), x_{I} \in \Omega_{0}\right\} \\
t_{n}^{e}=\min \left\{u^{e}\left(x_{I}\right)>t_{n-1}^{e}, x_{I} \in \Omega_{0}\right\} \quad \text { for } n \geq 2 .
\end{array}\right.
$$

We assume that $u^{e} \in C^{2}\left(\Omega_{0}\right)$ uniformly in e for e small enough, then there exist a discrete velocity $\hat{c}_{I}^{n-1, e}$, defined for $(I, n)$ such that $u\left(x_{I}\right)=t_{n}^{e}$, and a constant $C$ such that

$$
\left|\hat{c}_{I}^{n-1, e}-c\left(x_{I}, u\left(x_{I}\right)\right)-e\right| \leq O(\Delta x)
$$

and

$$
\theta^{\varepsilon, e}\left(x_{I}, t_{n}^{e}\right)=\theta^{e}\left(x_{I}, t_{n}^{e}\right)
$$

where $\theta^{\varepsilon, e}$ is the numerical solution of the GFMM algorithm with velocity $\hat{c}_{I}^{n, e}$ and with initial condition as in $(8.2)$.

To prove Theorem 8.1, we also need another version of the comparison principle Theorem 3.4 with weak assumptions on the velocities:

\section{Proposition 8.4 (Comparison principle for the $\theta^{\varepsilon}$ )}

Let $T>0$. Let two velocities $c_{u} \geq 0$ and $c_{v} \geq 0$. Given $\theta_{u, J}^{0}$ (resp. $\theta_{v, J}^{0}$ ) for all $J \in \mathbb{Z}^{N}$ and $u_{J \rightarrow K}^{0}$ for all $J \in \mathcal{U}_{u}^{0}(K)$ (resp. $v_{J \rightarrow K}^{0}$ for all $J \in \mathcal{U}_{v}^{0}(K)$ ), we assume that

$$
\begin{gathered}
t_{0}:=\sup _{J \in \mathcal{U}_{u}^{0}(K), K \in N B_{u}^{0}} u_{J \rightarrow K}^{0} \leq T, \\
\left(\text { resp. } s_{0}:=\sup _{J \in \mathcal{U}_{v}^{0}(K), K \in N B_{v}^{0}} v_{J \rightarrow K}^{0} \leq T\right)
\end{gathered}
$$

for a given $T>0$.

We also assume that the two velocities satisfy for all $(x, t) \in \mathbb{R}^{N} \times\left[\min \left(t_{0}, s_{0}\right), T-\Delta t\right]$

$$
\hat{c}_{v, I}^{m-1} \geq \sup _{s \in[-\Delta t, \Delta t], s_{m-1}+s \geq 0} c_{u}\left(x_{I}, s_{m-1}+s\right),
$$

for all $I \in \mathbb{Z}^{N}, m \in \mathbb{N}$ such that $I \in N A_{v}^{m}$.

If $C\left(t_{0}, s_{0}\right)$ and $\theta_{v}^{0} \succeq \theta_{u}^{0}$, then

$$
\theta_{v}^{\varepsilon}(x, t) \geq \theta_{u}^{\varepsilon}(x, t)
$$

for all $(x, t) \in \mathbb{R}^{N} \times\left[\max \left(t_{0}, s_{0}\right), T\right]$. 


\section{Proof of Proposition 8.4}

In fact, in the proof of Theorem 3.4, when the velocities are positive, the only point where we have to compare the velocities is in Lemma 5.6 and it is in fact sufficient to have an information on $c_{v, I}$ only when $I \in N A_{v}^{m}$ (i.e. for $\hat{c}_{v, I}^{m-1}$ )

\section{Proof of Theorem 8.1}

Let us denote by $u$ the solution of (8.1) such that $\theta(x, t)=1_{\{u(x)>t\}}-1_{\{u(x) \leq t\}}$. Given a mesh $\left\{x_{I}=\right.$ $\left.I \Delta x, I \in \mathbb{Z}^{n}\right\}$, we define a sequence of discrete increasing time $t_{n}$ in the following way

$$
\left\{\begin{array}{l}
t_{1}=\min \left\{u\left(x_{I}\right), x_{I} \in \Omega_{0}\right\} \\
t_{n}=\min \left\{u\left(x_{I}\right)>t_{n-1}, x_{I} \in \Omega_{0}\right\} \quad \text { for } n \geq 2 .
\end{array}\right.
$$

Then we consider $\theta^{\varepsilon, 0}(x, t)$ the projection of $\theta$ on the mesh, defined by

$$
\theta^{\varepsilon, 0}(x, t)=\theta\left(x_{I}, t_{n}\right) \text { if } x \in\left[x_{I}, x_{I}+\Delta x\right) \text { and } t \in\left[t_{n}, t_{n+1}\right) .
$$

In particular, we have

$$
\left|\theta^{\varepsilon, 0}-\theta\right|_{L^{\infty}\left((0, T) ; L^{1}\left(\mathbb{R}^{N}\right)\right)} \leq C(\Delta x+\Delta t)
$$

where $C$ is a constant (depending on $\|u\|_{C^{2}}$ ). Let us call $\theta^{\varepsilon}$ the numerical solution obtained by the GFFM algorithm and extended in the continuous space by (3.1). For $e>0$, we consider the discrete velocities $\hat{c}_{I}^{n-1,+e}$ and $\hat{c}_{I}^{n-1,-e}$ defined by Lemma 8.3 from Problem (8.2) with velocity $c+e$ and $c-e$ respectively. From (8.4), we have, for $e \geq C(\Delta x+\Delta t)$ and $C$ large enough

$$
\hat{c}_{I}^{n-1,+e} \geq \sup _{s \in[-\Delta t, \Delta t], t_{n-1}+s \geq 0} c\left(x_{I}, t_{n-1}+s\right), \quad \hat{c}_{I}^{n-1,-e} \leq \inf _{s \in[-\Delta t, \Delta t], t_{n-1}+s \geq 0} c\left(x_{I}, t_{n-1}+s\right)
$$

for $(I, n)$ such that $u^{e}\left(x_{I}\right)=t_{n}^{e}$.

Using the comparison principle Proposition 8.4, we then get

$$
\theta^{\varepsilon,+e} \geq \theta^{\varepsilon} \text { and } \theta^{\varepsilon,-e} \leq \theta^{\varepsilon} .
$$

By the comparison principle for equation (1.1), we have $\theta^{-e} \leq \theta \leq \theta^{e}$ and then by Lemma 8.3, the following inequality holds

$$
\theta^{\varepsilon,-e} \leq \theta^{\varepsilon, 0} \leq \theta^{\varepsilon,+e} .
$$

Choosing $e:=C(\Delta x+\Delta t)=O(|\varepsilon|)$, we can conclude that

$$
\begin{aligned}
\left|\theta^{\varepsilon}(\cdot, t)-\theta(\cdot, t)\right|_{L^{\infty}\left((0, T) ; L^{1}\left(\mathbb{R}^{N}\right)\right)} & \leq\left|\theta^{\varepsilon}-\theta^{\varepsilon, 0}\right|_{L^{\infty}\left((0, T) ; L^{1}\left(\mathbb{R}^{N}\right)\right)}+\left|\theta^{\varepsilon, 0}-\theta\right|_{L^{\infty}\left((0, T) ; L^{1}\left(\mathbb{R}^{N}\right)\right)} \\
& \leq\left|\theta^{\varepsilon,+e}(\cdot, t)-\theta^{\varepsilon,-e}(\cdot, t)\right|_{L^{\infty}\left((0, T) ; L^{1}\left(\mathbb{R}^{N}\right)\right)}+C(\Delta x+\Delta t) \\
& \leq K_{T}(\Delta x+\Delta t) .
\end{aligned}
$$

where $K_{T}$ is a positive constant. The last inequality follows by regularity property of equation (1.1) (see for instance [1]).

We now give the proof of Lemma 8.3:

\section{Proof of Lemma 8.3}

We define

$$
\hat{u}_{J}^{n, e}= \begin{cases}u^{e}\left(x_{J}\right) & \text { if } u^{e}\left(x_{J}\right) \leq t_{n}^{e} \\ +\infty & \text { otherwise }\end{cases}
$$

To construct the velocity $\hat{c}_{I}^{n-1, e}$, we solve (2.1) (where the unknown is the velocity $\hat{c}_{I}^{n-1, e}$ ) for $I$ such that $u^{e}\left(x_{I}\right)=t_{n}^{e}$. In particular, we have

$$
\hat{c}_{I}^{n-1, e}=c\left(x_{I}, t_{n}^{e}\right)+e+O(\Delta x)
$$

and by construction

$$
\theta^{\varepsilon, e}\left(x_{I}, t_{n}^{e}\right)=\theta^{e}\left(x_{I}, t_{n}^{e}\right)
$$

\section{Acknowledgements}

The author would like to thank E. Carlini and R. Monneau for fruitful discussions in the preparation of this paper. This work was supported by the ACI JC 1025 and the ANR MICA (2006-2009). 


\section{References}

[1] O. Alvarez, P. Cardaliaguet, and R. Monneau, Existence and uniqueness for dislocation dynamics with nonnegative velocity, Interfaces and Free Boundaries, 7 (2005), pp. 415-434.

[2] O. Alvarez, P. Hoch, Y. Le Bouar, and R. Monneau, Dislocation dynamics: short time existence and uniqueness of the solution, Archive for Rational Mechanics and Analysis, 85 (2006), pp. 371-414.

[3] G. Barles And P. E. Souganidis, Convergence of approximation schemes for fully nonlinear second order equations, Asymptotic Anal., 4 (1991), pp. 271-283.

[4] E. Carlini, M. Falcone, N. Forcadel, and R. Monneau, Convergence of a generalized fast marching method for a non-convex eikonal equation, To appear in SIAM J. Num. Anal., (2006).

[5] E. Carlini, N. Forcadel, and R. Monneau, Generalized fast marching method for dislocation dynamics. Work in progress.

[6] M. G. Crandall And P.-L. Lions, Two approximations of solutions of Hamilton-Jacobi equations, Math. Comp., 43 (1984), pp. 1-19.

[7] M. FAlCone, The minimum time problem and its applications to front propagation, in Motion by mean curvature and related topics (Trento, 1992), de Gruyter, Berlin, 1994, pp. 70-88.

[8] J. A. Sethian, A fast marching level set method for monotonically advancing fronts, Proc. Nat. Acad. Sci. U.S.A., 93 (1996), pp. 1591-1595.

[9] — Level set methods and fast marching methods, vol. 3 of Cambridge Monographs on Applied and Computational Mathematics, Cambridge University Press, Cambridge, second ed., 1999. Evolving interfaces in computational geometry, fluid mechanics, computer vision, and materials science.

[10] J. N. TsitsikLIs, Efficient algorithms for globally optimal trajectories, IEEE Trans. Automat. Control, 40 (1995), pp. 1528-1538.

[11] A. Vladimirsky, Static pdes for time-dependent control problems, Interfaces and Free Boundaries, 8 (2006), pp. 281-300. 